Ronaldo Mendes Evaristo

\title{
Métodos de Reamostragem de Séries Temporais Baseados em Wavelets
}

\author{
Dissertação apresentada à Escola Po- \\ litécnica da Universidade de São Paulo \\ para obtenção do Título de Mestre em \\ Engenharia Elétrica.
}


Ronaldo Mendes Evaristo

\title{
Métodos de Reamostragem de Séries Temporais Baseados em Wavelets
}

\author{
Dissertação apresentada à Escola Po- \\ litécnica da Universidade de São Paulo \\ para obtenção do Título de Mestre em \\ Engenharia Elétrica. \\ Área de concentração: \\ Sistemas Eletrônicos \\ Orientador: \\ Prof. Dr. Luiz Antonio Baccalá
}




\section{Ficha Catalográfica}

Evaristo, Ronaldo Mendes

Métodos de Reamostragem de Séries Temporais Baseados em $W a$ velets. São Paulo, 2010. 53 p.

Dissertação (Mestrado) - Escola Politécnica da Universidade de São Paulo. Departamento de Telecomunicações e Controle.

1. Análise de Séries Temporais. 2. Análise de Ondaletas. 3. Análise Espectral. I. Universidade de São Paulo. Escola Politécnica. Departamento de Telecomunicações e Controle. II. Título. 
Aos meus pais e familiares. 


\section{Agradecimentos}

À Deus, pela companhia em todos os momentos.

Ao Prof. Dr. Luiz Antonio Baccalá meu agradecimento pelo acolhimento, orientação, apoio e confiança depositada, indispensáveis para a concretização deste trabalho.

Ao Prof. Dr. Celso Volpe meu agradecimento especial pelas enormes contribuições intelectuais desde o tempo de graduação sem as quais não seria possível iniciar este trabalho.

Ao colega de laboratório, Bruno Augusto Angélico, pela prestatividade e colaboração.

Aos demais colegas e professores do LCS, pelas valiosas discussões.

Aos professores Dr. Alexandre Rocco, Dr. Heraldo Silveira Barbuy, MsC. Salvador José Troise pelo constante acompanhamento durante este trabalho.

À Universidade Santa Cecília pela crença sobre mim depositada.

À Universidade de São Paulo e a Escola Politécnica pelo ambiente, estrutura e seriedade.

Ao CNPq pelo apoio financeiro. 


\section{Resumo}

Neste texto são revisados métodos de reamostragem de séries temporais discretas baseados em wavelets, como alternativas às abordagens clássicas, feitas nos domínios do tempo e da frequência. Tais métodos, conhecidos na literatura como wavestrap e wavestrapping fazem uso, respectivamente, das transformadas wavelet discreta (DWT) e wavelet packet discreta (DWPT). Existem poucos resultados sobre a aplicação da DWPT, de forma que este texto pode ser considerado uma contribuição.

Aqui mostra-se também, a superioridade do wavestrapping sobre o wavestrap quando aplicados na estimação da densidade espectral de potência de séries temporais sintéticas geradas a partir de modelos autoregressivos. Tais séries possuem uma particularidade interessante que são picos, geralmente acentuados, em sua respresentação espectral, de tal forma que grande parte dos métodos clássicos de reamostragem apresentam resultados viesados quando aplicados a estes casos. 


\section{Abstract}

This paper reviews resampling methods based on wavelets as an alternative to the classic approaches which are, made in the time and frequency domains. These methods, known in the literature as wavestrap and wavestrapping, make use, respectively, of the discrete wavelet transform (DWT) and of the discrete wavelet packet transform (DWPT). Since only few results are avaliable when the DWPT is applied, this text can be considered a contribution to the subject.

Here we, also show the superiority of wavestrapping over wavestrap when they are applied to the estimation of power spectral densities of the synthetic time series generated from autoregressive models. These series have an interesting feature that are sharp peaks in their spectral representation, so that most of the traditional methods of resampling lead to biased results. 


\title{
Sumário
}

\section{Lista de Figuras}

\author{
Lista de Abreviaturas
}

1 Introdução 1

1.1 Estrutura da Dissertação . . . . . . . . . . . . . . . . . . 2

I Fundamentação Teórica 3

2 Conceitos Básicos de Reamostragem 4

2.1 Motivação . . . . . . . . . . . . . . . . . . . . . 4

2.2 Procedimento de Bootstrap . . . . . . . . . . . . . . . 4

2.3 Reamostragem de Séries Temporais . . . . . . . . . . . . . 6

2.3.1 Reamostragem de Séries Temporais Aleatórias . . . . . . . 7

2.3.2 Reamostragem de Séries Temporais Autoregressivas . . . . 9

2.4 Outros Métodos de Reamostragem de Séries Correlacionadas . . . 10

3 Decomposição Wavelet 12

3.1 Motivação . . . . . . . . . . . . . . . . . . . . . 12

3.2 Transformada Wavelet Discreta de Tempo Discreto . . . . . . . . 12

3.3 Transformada Wavelet Packet Discreta de Tempo Discreto . . . . 15

II Análise Experimental 18

4 Reamostragem Baseada em Wavelets 19

4.1 Procedimentos de Wavestrap e Wavestrapping . . . . . . . . . . 19 
4.2 Cenários de Estudo . . . . . . . . . . . . . . . . . . . . . 21

4.2.1 Considerações sobre a Ordem do Filtro Wavelet . . . . . . 22

4.2.2 Considerações sobre o Número de Níveis na Decomposição 32

$\begin{array}{ll}\text { IIIConclusões } & 37\end{array}$

5 Conclusões e Trabalhos Futuros $\quad 38$

5.1 Trabalhos Futuros . . . . . . . . . . . . . . . . . . 39

Anexo A - Banco de Filtros: Considerações sobre Reconstrução 40

Anexo B - Decomposição Wavelet: Construção da Matriz $\mathcal{W} \quad 42$

$\begin{array}{ll}\text { Anexo C - Códigos MATLAB } & 47\end{array}$

$\begin{array}{ll}\text { Referências } & 51\end{array}$

Apêndice A - Artigo Publicado 53 


\section{Lista de Figuras}

2.1 Diagrama do procedimento de bootstrap. . . . . . . . . . . . 5

2.2 Procedimento 2 - (a) Histograma das estimativas bootstrap $\mu_{i}^{*}, i=$ $1, \cdots, 5000 ;$ (b) Distribuição empírica $F^{*}$ do estimador $\hat{\mu}$. . . . . . . 5

2.3 (a) Série temporal aleatória, $N=256$; (b) Correlograma para $k=100 . \quad 8$

2.4 Vizualização de $x(n)$ (autoregressivo) como saída de um sistema. . . . 9

2.5 (a) Série temporal tomada de um modelo autoregressivo de ordem 1 sendo $A(z)=1-0,95 z^{-1}$ e $N=256$; (b) Correlograma de $x(n)$ para $k=100 ;$ (c) Reamostragem $x^{*}(n)$ de $x(n)$; (d) Correlograma de $x^{*}(n)$.

2.6 Diagrama do moving block bootstrap em que são escolhidos trechos de comprimento $l$ do sinal para serem reamostrados de acordo com os procedimentos 3 e $4 \ldots \ldots \ldots \ldots 11$

3.1 DWT - Decomposição para $J_{0}=3 \ldots \ldots 13$

3.2 DWT - Reconstrução para $J_{0}=3 \ldots \ldots$. . . . . . . . . 13

3.3 DWT - Relação entre os coeficientes e suas respectivas bandas espectrais. 14

3.4 DWPT - Decomposição para $J_{0}=3 \ldots \ldots$. . . . . . . . . 16

3.5 DWPT - Reconstrução para $J_{0}=3 \ldots \ldots$. . . . . . . . . . . . 16

3.6 DWPT - Relação entre os coeficientes e suas respectivas bandas espectrais. 17

3.7 Caminho alternativo para reconstrução DWPT. . . . . . . . . . . . . 17

4.1 Diagrama da reamostragem baseada em wavelets. . . . . . . . . . . . 19

4.2 Geração de uma série temporal por um filtro AR. . . . . . . . . . . . 21

4.3 Respostas em frequência normalizadas dos filtros AR considerados no estudo.

4.4 Resposta em frequência normalizada dos filtros de decomposição DWT em 4 níveis utilizando as wavelets de Daubechies de ordens: (a) 80; (b) 16 . 
4.5 (a) Primeira série temporal $\left(x_{1}(n)\right)$; (b) Seu espectro de potência normalizado $\left(\hat{S}_{1 N}\left(e^{j \omega}\right)\right) \ldots \ldots \ldots \ldots \ldots$

4.6 Autocorrelações dos coeficientes da decomposição DWT de $x_{1}(n)$ em 4 níveis utilizando filtros Daubechies de ordem 8. . . . . . . . . . . . . 24

4.7 Autocorrelações dos coeficientes da decomposição DWPT de $x_{1}(n)$ em 4 níveis utilizando filtros Daubechies de ordem 8. . . . . . . . . . . . 24

4.8 Resposta $S_{1 N}\left(e^{j \omega}\right)$ sobreposta à resposta dos filtros Daubechies, na decomposição DWT de 4 níveis, de ordem (a) 8; (b) 32. . . . . . . . . .

4.9 Estimativas de $S_{1 N}\left(e^{j \omega}\right)$ utilizando decomposições em 4 níveis com filtros Daubechies de ordem 8 dadas pelos procedimentos de (a) wavestrap; (b) wavestrapping. . . . . . . . . . . . . . . .

4.10 Estimativas de $S_{1 N}\left(e^{j \omega}\right)$ utilizando decomposições em 4 níveis com filtros Daubechies de ordem 32 dadas pelos procedimentos de (a) wavestrap; (b) wavestrapping. . . . . . . . . . . . . . . .

4.11 (a) Segunda série temporal $\left(x_{2}(n)\right)$; (b) Seu espectro de potência normalizado $\left(\hat{S}_{2 N}\left(e^{j \omega}\right)\right) \ldots \ldots \ldots \ldots \ldots$

4.12 Autocorrelações dos coeficientes da decomposição DWT de $x_{2}(n)$ em 4 níveis utilizando filtros Daubechies de ordem 16. . . . . . . . .

4.13 Autocorrelações dos coeficientes da decomposição DWPT de $x_{2}(n)$ em 4 níveis utilizando filtros Daubechies de ordem 16. . . . . . . . .

4.14 Resposta $S_{2 N}\left(e^{j \omega}\right)$ sobreposta à resposta dos filtros Daubechies, na decomposição DWT de 4 níveis, de ordem (a) 16; (b) 80....... .

4.15 Estimativas de $S_{2 N}\left(e^{j \omega}\right)$ utilizando decomposições em 4 níveis com filtros Daubechies de ordem 16 dadas pelos procedimentos de (a) wavestrap; (b) wavestrapping. . . . . . . . . . . . . . . . . .

4.16 Estimativas de $S_{2 N}\left(e^{j \omega}\right)$ utilizando decomposições em 4 níveis com filtros Daubechies de ordem 80 dadas pelos procedimentos de (a) wavestrap; (b) wavestrapping. . . . . . . . . . . . . . . . . .

4.17 (a) Terceira série temporal $\left(x_{3}(n)\right)$; (b) Seu espectro de potência normalizado $\left(\hat{S}_{3 N}\left(e^{j \omega}\right)\right) \ldots \ldots \ldots \ldots \ldots$

4.18 Autocorrelações dos coeficientes da decomposição DWT de $x_{3}(n)$ em 4 níveis utilizando filtros Daubechies de ordem 20. . . . . . . . . . 
4.19 Autocorrelações dos coeficientes da decomposição DWPT de $x_{3}(n)$ em 4 níveis utilizando filtros Daubechies de ordem 20. . . . . . . . . . . 30

4.20 Resposta $S_{3 N}\left(e^{j \omega}\right)$ sobreposta à resposta dos filtros Daubechies, na decomposição DWT de 4 níveis, de ordem (a) 20; (b) 80. . . . . . . . . 3

4.21 Estimativas de $S_{3 N}\left(e^{j \omega}\right)$ utilizando decomposições em 4 níveis com filtros Daubechies de ordem 20 dadas pelos procedimentos de (a) wavestrap; (b) wavestrapping. . . . . . . . . . . . . . . .

4.22 Estimativas de $S_{3 N}\left(e^{j \omega}\right)$ utilizando decomposições em 4 níveis com filtros Daubechies de ordem 80 dadas pelos procedimentos de (a) wavestrap; (b) wavestrapping. . . . . . . . . . . . . . . .

4.23 Autocorrelações dos coeficientes da decomposição DWT de $x_{2}(n)$ em 5 níveis utilizando filtros Daubechies de ordem 80. . . . . . . . .

4.24 Autocorrelações dos coeficientes da decomposição DWPT de $x_{2}(n)$ em 5 níveis utilizando filtros Daubechies de ordem 80. . . . . . . . . .

4.25 Resposta $S_{2 N}\left(e^{j \omega}\right)$ sobreposta à resposta dos filtros Daubechies de ordem 80, na decomposição em 5 níveis utilizando a (a) DWT; (b) DWPT. 34

4.26 Estimativas de $S_{2 N}\left(e^{j \omega}\right)$ utilizando decomposições em 5 níveis com filtros Daubechies de ordem 80 dadas pelos procedimentos de (a) wavestrap; (b) wavestrapping. . . . . . . . . . . . . . . . 34

4.27 Autocorrelações dos coeficientes da decomposição DWT de $x_{3}(n)$ em 5 níveis utilizando filtros Daubechies de ordem 80. . . . . . . . . . 35

4.28 Autocorrelações dos coeficientes da decomposição DWPT de $x_{3}(n)$ em 5 níveis utilizando filtros Daubechies de ordem $80 \ldots \ldots$. . . . . .

4.29 Resposta $S_{3 N}\left(e^{j \omega}\right)$ sobreposta à resposta dos filtros Daubechies de ordem 80, na decomposição em 5 níveis utilizando a (a) DWT; (b) DWPT. 36

4.30 Estimativas de $S_{3 N}\left(e^{j \omega}\right)$ utilizando decomposições em 5 níveis com filtros Daubechies de ordem 80 dadas pelos procedimentos de (a) wavestrap; (b) wavestrapping. . . . . . . . . . . . . 36

A.1 Banco de filtros para decomposição wavelet. . . . . . . . . . . . . . . 40

B.1 Sinal $x(n)$ de comprimento $N=16 \ldots \ldots . \ldots . \ldots 42$

B.2 Árvore matricial para a DWT com $J_{0}=3 \ldots \ldots \ldots$. . . . . . . 43

B.3 Árvore matricial inversa para a DWT com $J_{0}=3 \ldots \ldots \ldots$. . . . . . 45 
B.4 Árvore matricial para a DWPT com $J_{0}=3 \ldots \ldots$. . . . . . . . . 46 


\title{
Lista de Abreviaturas
}

\author{
AR Autoregressive \\ CBB Circular Block Bootstrap \\ DFT Discrete Fourier Transform \\ DSP Digital Signal Processor \\ DWT Discrete Wavelet Transform \\ DWPT Discrete Wavelet-Packet Transform \\ EEG Eletroencefalograma \\ fMRI Functional Magnetic Resonance Imaging \\ MA Moving Average \\ MBB Moving Block Bootstrap \\ NBB Nonoverlapping Block Bootstrap \\ SBB Stationary Block Bootstrap \\ STFT Short-Time Fourier Transform
}




\section{Introdução}

Idealmente, quando há interesse em estimar certos parâmetros de um processo estocástico, são necessárias várias realizações do mesmo para que se possa determinar o grau de confiabilidade das estimativas produzidas. Ocorre que nem sempre é possível realizar um experimento mais de uma vez e ainda, na grande maioria dos casos, o número de observações de uma realização é pequeno, gerando assim, a necessidade dos métodos de reamostragem. Tais métodos surgiram das idéias básicas de bootstrap, introduzidas por Efron [1], e cabem a eles gerar realizações hipotéticas (dados reamostrados) de um determinado experimento com base em algumas realizações iniciais fornecidas.

Por outro lado, os métodos baseados em bootstrap, exigem que os dados a serem reamostrados sejam provenientes de variáveis aleatórias independentes e identicamente distribuídas, o que limita sua aplicação direta sobre séries temporais, em que as observações geralmente apresentam algum tipo de correlação. Isto têm motivado muitos pesquisadores a buscarem ferramentas que, previamente, descorrelacionem as amostras das séries para uma posterior aplicação do procedimento de bootstrap. Este descorrelacionamento prévio, chamado de prewhitening [2], sugere o uso de transformadas como são os casos da transformada wavelet discreta (DWT) e a transformada discreta de Fourier (DFT). O método que utiliza a DFT foi revisto recentemente em [3] e aqui é feita uma avaliação dos que utilizam wavelets.

Com a introdução do algoritmo piramidal, feita por Mallat [4], permitindo o cálculo eficiente da DWT, certas propriedades vieram à tona. Uma destas propriedades, discutida por vários autores [5, 6, 7, 8, 9], diz que seus coeficientes, sob certas condições, tendem a ser descorrelacionados, o que levou Bullmore et. al. $[2,10]$ a introduzir a reamostragem baseada em wavelets, conhecida atualmente como wavestrap.

As condições sob as quais os coeficientes wavelet são descorrelacionados ainda não foram bem estabelecidas e ainda vêm sendo investigadas. Dentre elas, a que provavelmente limita grande parte dos métodos de reamostragem, é a presença 
de picos nas representações espectrais das séries em estudo.

Alguns autores $[2,5]$ mostraram que o wavestrap funciona bem para modelos cujas densidades espectrais de potência são suaves, por exemplo, aquelas provenientes de modelos de média móvel (MA). Contudo, quando o espectro possui picos muito acentuados, que é o caso dos modelos autoregressivos (AR) com polos próximos da circunferência unitária, os métodos baseados na DWT apresentam viés estatístico dependendo da forma como são aplicados.

Nesta dissertação fazemos uma revisão dos métodos baseados na DWT e estudamos um método que usa a transformada wavelet packet discreta (DWPT) como alternativa. Na realidade, o uso da DWPT é uma contribuição do presente trabalho.

\subsection{Estrutura da Dissertação}

Em sequência a esta introdução, o Capítulo 2 apresenta uma revisão das idéias básicas associadas aos conceitos de reamostragem. Além disto, o capítulo apresenta também uma motivação para o uso dos filtros wavelet como modo de reduzir algumas limitações presentes nos métodos clássicos de reamostragem.

Outro aspecto teórico importante (Capítulo 3), diz respeito à decomposição wavelet nas duas diferentes maneiras consideradas aqui, que são as DWT e DWPT.

Para validar o uso das wavelets no contexto do estudo de reamostragem é realizada uma investigação exploratória (Capítulo 4) envolvendo sinais correlacionados, provenientes de modelos autoregressivos, utilizando as duas transformadas em questão.

Alguns anexos são apresentados com o intuito de situar o leitor pouco familiarizado com o assunto. No anexo A são feitas algumas considerações a respeito de reconstrução perfeita, conceito associado a bancos de filtros. No anexo B são apresentados exemplos numéricos de decomposição wavelet, baseados na teoria presente em [11] e, finalmente, como ponto de vista prático e a título de contribuição ao grupo de pesquisa, são apresentados, no anexo $\mathrm{C}$, alguns scripts em MATLAB que foram utilizados durante o trabalho para realizar decomposições wavelets. Um artigo sobre este estudo, apresentado em congresso, consta do apêndice $\mathrm{A}$, encerrando o texto. 
Parte I

Fundamentação Teórica 


\section{Conceitos Básicos de Reamostragem}

\subsection{Motivação}

Quando há interesse em estimar um certo parâmetro $\theta(X)$ de uma variável aleatória $X$, através de um estimador $\hat{\theta}$ que utiliza observações $x(0), \cdots, x(N-1)$ de $X$, são necessárias estatísticas que permitam avaliar o grau de confiabilidade das estimativas produzidas como, por exemplo, intervalos de confiança. Contudo, tais intervalos dependem da distribuição $F$ de $\hat{\theta}$, que por sua vez, depende de várias realizações do experimento para ser estimada. Ocorre que nem sempre é possível realizar um experimento mais de uma vez e para isso surgiram os métodos de reamostragem baseados em sua maioria na idéia básica de bootstrap, proposta inicialmente por Bradley Efron [1], foco desta seção.

\section{$2.2 \quad$ Procedimento de Bootstrap}

A rotina de bootstrap, ilustrada na figura 2.1, é apresentada no procedimento 1 e tem por objetivo gerar realizações hipotéticas de um determinado experimento com base em algumas realizações do mesmo.

Procedimento 1 Bootstrap

Entrada: Sejam $N$ observações da variável aleatória $X$ dispostas em forma de vetor

$$
\mathbf{X}=[x(0) \cdots x(N-1)]^{T} .
$$

1. Escolha aleatoriamente, com reposição e igual probabilidade, $N$ observações $x(n)$ de $\mathbf{X}$ e monte o vetor reamostrado $\mathbf{X}^{*}=\left[x^{*}(0) \cdots x^{*}(N-1)\right]^{T}$ de $\mathbf{X}$.

2. Calcule uma estimativa $\hat{\theta}\left(\mathbf{X}^{*}\right)$ para o parâmetro $\theta(X)$ por meio do estimador $\hat{\theta}$.

3. Repita $B$ vezes os itens 1 e 2 e obtenha a distribuição $F^{*}$ de $\hat{\theta}$ que se aproxima de $F$, a verdadeira distribuição de $\hat{\theta}$, conforme $B$ aumenta [12].

4. Utilize $F^{*}$ para avaliar o grau de confiabilidade de $\hat{\theta}$. 
No procedimento 2 é apresentado um exemplo de aplicação do bootstrap para o estabelecimento de um intervalo de confiança de $95 \%$ em torno da média de uma variável aleatória $X$ cujas observações são extraídas de

$$
x(n)=5+w(n),
$$

com $w(n) \sim \mathcal{N}(0,1)$, utilizando o estimador

$$
\hat{\mu}=\frac{1}{N} \sum_{n=0}^{N-1} x(n),
$$

ou seja, será determinado um intervalo $\left[\hat{\mu}_{I}, \hat{\mu}_{S}\right]$ para a média $\mu=5$ de $X$ tal que,

$$
P\left(\hat{\mu}_{I} \leq \mu \leq \hat{\mu}_{S}\right)=1-\alpha
$$

$\operatorname{com} \alpha=0,05$.

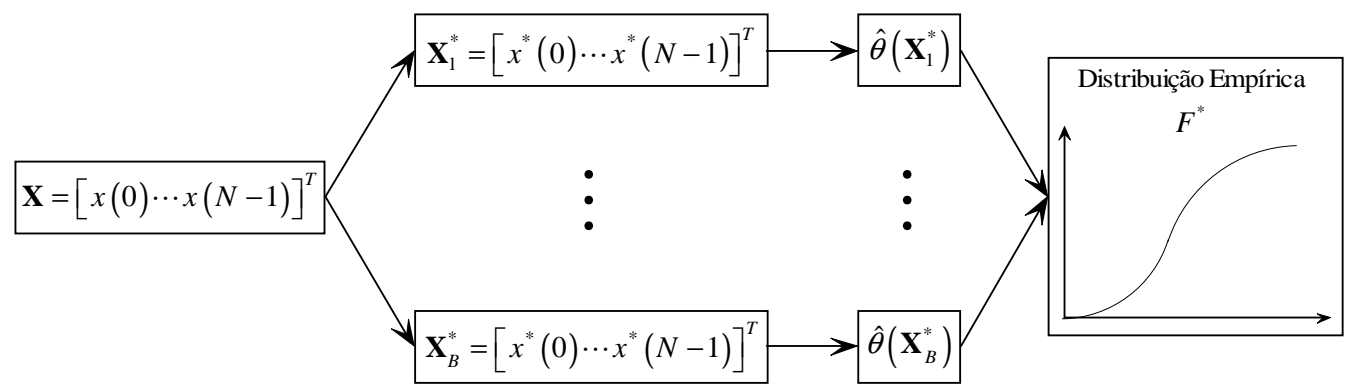

Figura 2.1: Diagrama do procedimento de bootstrap.

Neste exemplo é interessante observar que a confiabilidade do estimador $\hat{\mu}$ depende de quanto se sabe, a priori, sobre $w(n)[3]$. Por exemplo, o fato de $w(n)$ ser normalmente distribuído com média nula e variância unitária garante que o estimador $\hat{\mu}$ seja não viesado e que sua variância possa ser estimada com base nas observações, permitindo desta forma, o estabelecimento do intervalo de confiança desejado.

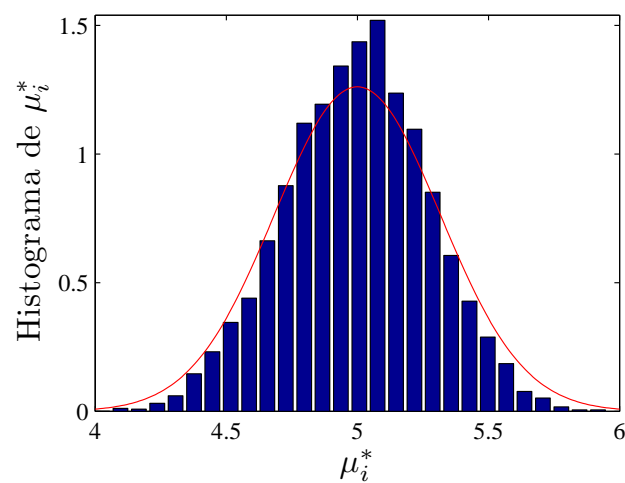

(a)

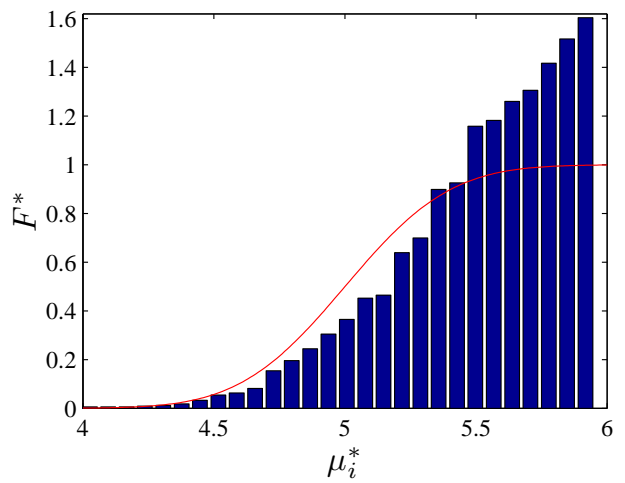

(b)

Figura 2.2: Procedimento 2 - (a) Histograma das estimativas bootstrap $\mu_{i}^{*}$, $i=1, \cdots, 5000 ;$ (b) Distribuição empírica $F^{*}$ do estimador $\hat{\mu}$. 
Procedimento 2 Bootstrap: Intervalo de Confiança para a Média

Entrada: Sejam 10 observações da variável $X$ tomadas por meio da equação 2.1,

$$
\mathbf{X}=\left[\begin{array}{llllllllll}
5,56 & 6,25 & 4,58 & 3,54 & 4,05 & 6,39 & 4,03 & 4,50 & 5,51 & 5,35
\end{array}\right]^{T} .
$$

1. Escolha aleatoriamente, com reposição e igual probabilidade, 10 observações de $\mathbf{X}$ e monte o vetor reamostrado

$$
\mathbf{X}^{*}=\left[\begin{array}{llllllllll}
5,56 & 5,56 & 6,25 & 4,58 & 6,25 & 4,50 & 4,58 & 5,56 & 4,58 & 4,50
\end{array}\right]^{T} .
$$

2. Calcule uma estimativa para a média $\hat{\mu}\left(\mathbf{X}^{*}\right)=5,29$ por meio do estimador $\hat{\mu}$ apresentado na equação 2.2 .

3. Repita, por exemplo, $B=5000$ vezes os itens 1 e 2 e obtenha $\hat{\mu}\left(\mathbf{X}_{1}^{*}\right) \cdots \hat{\mu}\left(\mathbf{X}_{5000}^{*}\right)$, cujo histograma é mostrado na figura $2.2(\mathrm{a})$.

4. Ordene as estimativas $\hat{\mu}\left(\mathbf{X}_{i}^{*}\right)$ de forma crescente

$$
\hat{\mu}_{1}^{*} \leq \cdots \leq \hat{\mu}_{I}^{*} \leq \cdots \leq \hat{\mu}_{S}^{*} \leq \cdots \leq \hat{\mu}_{5000}^{*}
$$

e construa a distribuição empírica $F^{*}$ de $\hat{\mu}$, ilustrada na figura 2.2(b).

5. Obtenha o intervalo de confiança $(1-\alpha) 100 \%$ calculando os índices $I$ e $S$ da seguinte forma $[13,14,15]$

$$
I=\left\lfloor B \frac{\alpha}{2}\right\rfloor \quad \text { e } \quad S=B-I+1,
$$

ou seja,

$$
I=\left\lfloor 5000 \frac{0,05}{2}\right\rfloor=125 \quad \text { e } \quad S=5000-125+1=4876 .
$$

Saída: $\left[\hat{\mu}_{I}^{*}, \hat{\mu}_{S}^{*}\right]=[4,48 ; 5,60]$.

É importante notar também que no procedimento 2 as observações $x(n)$ são descorrelacionadas devido a $w(n)$ ser uma gaussiana. Esta é uma exigência que torna o bootstrap limitado, visto que a maioria dos dados encontrados na prática possuem algum tipo de dependência, como é o caso das séries temporais. Isto vêm motivando os pesquisadores a buscarem métodos para reamostrar observações dependentes, sendo que alguns são discutidos na próxima seção dando certo enfoque à reamostragem de séries temporais provenientes de modelos $\mathrm{AR}$ devido a sua aplicabilidade em Neurociência, interesse futuro para a aplicação deste estudo.

\subsection{Reamostragem de Séries Temporais}

Uma série temporal é uma sequência de observações $\{x(n): n=0, \cdots, N-1\}$, em geral colocadas na forma de vetor

$$
\mathbf{x}=[x(0) \cdots x(N-1)]^{T},
$$


tomadas a intervalos regulares de tempo onde cada observação provém de uma variável aleatória com distribuição, em geral, desconhecida [16]. Sendo assim, é possível pensar em uma série temporal $x(n)$ como sendo uma realização (função amostra) de um processo estocástico $\mathbf{x}(n)$.

\subsubsection{Reamostragem de Séries Temporais Aleatórias}

Uma das características mais importantes de uma série temporal é a dependência entre observações vizinhas, avaliada de acordo com os coeficientes de autocorrelação amostral ${ }^{1}$

$$
r_{k}(\mathbf{x})=\frac{\sum_{n=0}^{N-k-1}\left[x(n)-\hat{\mu}_{\mathbf{x}}\right]\left[x(n+k)-\hat{\mu}_{\mathbf{x}}\right]}{\sum_{n=0}^{N-1}\left[x(n)-\hat{\mu}_{\mathbf{x}}\right]^{2}}
$$

sendo

$$
\hat{\mu}_{\mathbf{x}}=\frac{1}{N} \sum_{n=0}^{N-1} x(n) .
$$

Um gráfico com os primeiros $k$ coeficientes de autocorrelação como função de $k$, chamado correlograma, é uma importante ferramenta para a identificação de aleatoriadade em uma série temporal.

Desta forma, supondo que as observações do vetor 2.3 sejam provenientes de variáveis aleatórias independentes e identicamente distribuídas, é possível mostrar que os coeficientes $r_{k}(\mathbf{x})$ são assintoticamente normalmente distribuídos com média e variância dadas por [17]

$$
E\left(r_{k}(\mathbf{x})\right)=-\frac{1}{N} \quad \text { e } \quad \operatorname{Var}\left(r_{k}(\mathbf{x})\right)=\frac{1}{N}
$$

Isto permite o estabelecimento de limites de confiança de $95 \%$ sobre o correlograma que indicam a presença de aleatoriedade. Tais limites são [17]

$$
-\frac{1}{N} \pm \frac{1,96}{\sqrt{N}}
$$

em geral aproximados por,

$$
\pm \frac{1,96}{\sqrt{N}}
$$

Na figura 2.3 é apresentada uma série temporal aleatória $x(n) \operatorname{com} N=256$, assim como seu correlograma para as 100 primeiras defasagens. Neste caso, é

\footnotetext{
${ }^{1}$ Os coeficientes de autocorrelação dados pela equação 2.4 valem para $N$ grande.
} 


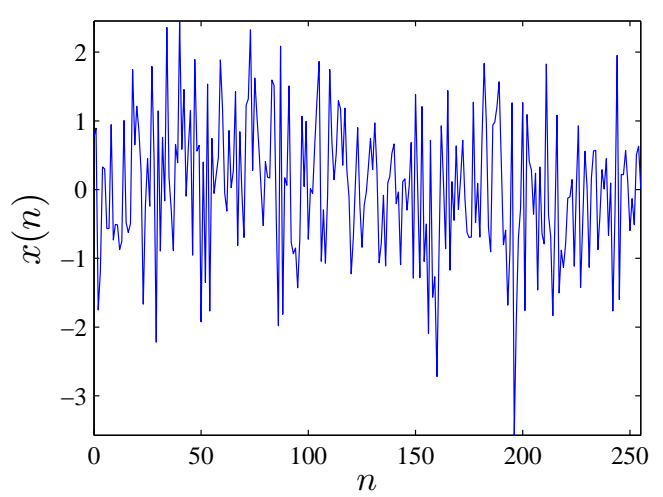

(a)

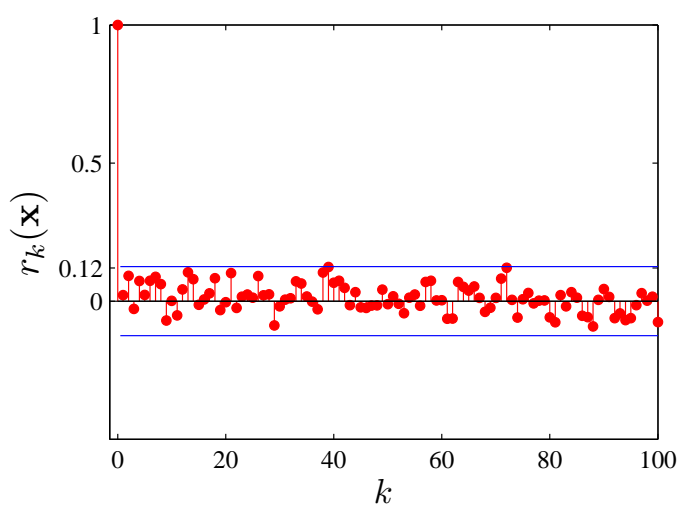

(b)

Figura 2.3: (a) Série temporal aleatória, $N=256$; (b) Correlograma para $k=100$.

possível notar que as autocorrelações não ultrapassam os limites de confiança \pm 0.12 , calculados por meio de 2.6 , o que permite afirmar que essa série é descorrelacionada.

Para estes casos são apresentados nos procedimentos 3 e 4 dois métodos de reamostragem baseados na rotina de bootstrap.

Procedimento 3 Reamostragem para Séries Aleatórias: Com reposição

Entrada: Série temporal $\mathbf{x}=[x(0) \cdots x(N-1)]^{T}$.

1. Escolha aleatoriamente, com reposição e igual probabilidade, $N$ observações $x(n)$ de $\mathbf{x}$ e monte a série reamostrada $\mathbf{x}^{*}=\left[x^{*}(0) \cdots x^{*}(N-1)\right]^{T}$ de $\mathbf{X}$.

2. Repita o item 1 e obtenha $B$ versões reamostradas de $\mathbf{x}$.

3. Utilize as séries reamostradas como realizações hipotéticas do processo $\mathbf{X}(n)$ e estime as estatísticas de interesse.

\section{Procedimento 4 Reamostragem para Séries Aleatórias: Sem reposição}

Entrada: Série temporal $\mathbf{x}=[x(0) \cdots x(N-1)]^{T}$.

1. Escolha aleatoriamente, sem reposição e igual probabilidade, $N$ observações $x(n)$ de $\mathbf{x}$ e monte a série reamostrada $\mathbf{x}^{*}=\left[x^{*}(0) \cdots x^{*}(N-1)\right]^{T}$ de $\mathbf{X}$.

2. Repita o item 1 e obtenha $B$ versões reamostradas de $\mathbf{x}$.

3. Utilize as séries reamostradas como realizações hipotéticas do processo $\mathbf{X}(n)$ e estime as estatísticas de interesse.

É interessante notar que a diferença entre os dois reside simplesmente no fato de como a escolha entre as observações é feita, ou seja, se é feita com ou sem reposição ou por meio de permutação. 


\subsubsection{Reamostragem de Séries Temporais Autoregressivas}

O presente trabalho por sua vez, é focado em séries temporais oriundas de modelos AR, ou seja, séries do tipo

$$
x(n)=\sum_{i=1}^{p} \alpha_{i} x(n-i)+w(n),
$$

com $w(n) \sim \mathcal{N}(0,1)$, que podem ser modeladas utilizando notação mista [18] de acordo com

$$
x(n)=\frac{1}{\left[1-\sum_{i=1}^{p} \alpha_{i} z^{-i}\right]} w(n)=\frac{1}{A(z)} w(n) .
$$

Com esta notação é possível olhar para uma série temporal gerada a partir de um modelo autoregressivo como sendo a saída de um sistema estável ${ }^{2}$ com função de transferência $\frac{1}{A(z)}$ quando na entrada temos ruído branco, conforme mostra a figura 2.4

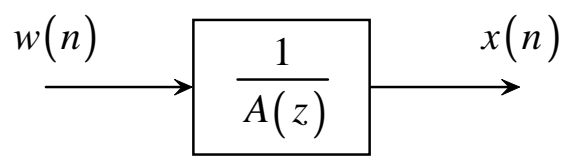

Figura 2.4: Vizualização de $x(n)$ (autoregressivo) como saída de um sistema.

Considerando, por exemplo,

$$
A(z)=1-0,95 z^{-1}
$$

e determinando $x(n)$ a partir da figura 2.4 para $N=256$ temos como resultado a série mostrada na figura 2.5(a), assim como seu correlograma na figura 2.5(b).

Aqui, se for aplicada a reamostragem de acordo com os procedimentos 3 e 4 tem-se um resultado viesado como o mostrado nas figuras 2.5 (c) e (d), que se deve a $x(n)$ possuir as observações correlacionadas.

Sendo assim, para que a reamostragem baseada em bootstrap possa ser aplicada sobre estas séries temporais é necessário primeiramente remover, de forma reversível, a correlação entre as observações. Este procedimento é conhecido na literatura como pre-whitening [2] e pode ser alcançado pela aplicação de transformadas; neste texto discutem-se a DWT e a DWPT. Outras ferramentas de pre-whitening como, por exemplo, a DFT, estão ilustradas em [3, 19] e um estudo comparativo entre a DWT e DFT em [19, 20], cujos autores aplicam os

\footnotetext{
${ }^{2} \mathrm{O}$ sistema é estável sempre que as raízes de $A(z)$ estiverem dentro da circunferência unitária no plano z.
} 


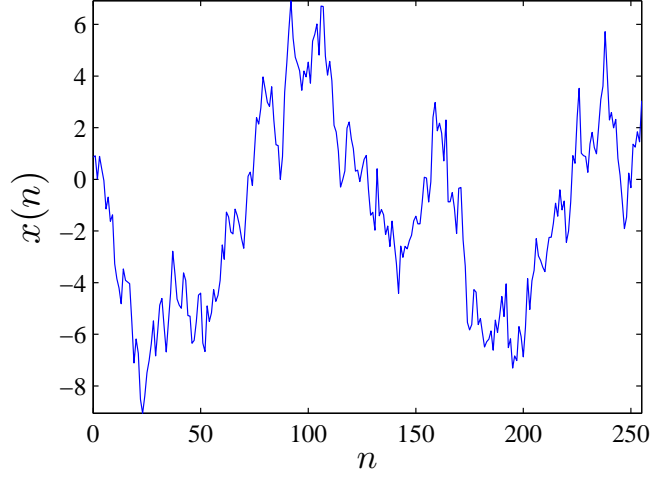

(a)

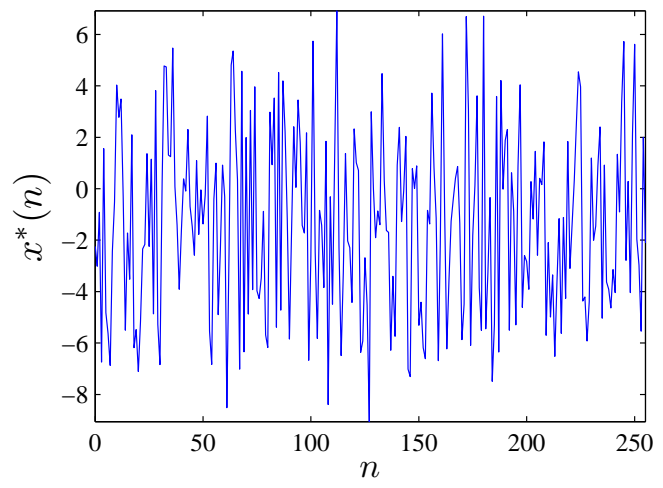

(c)

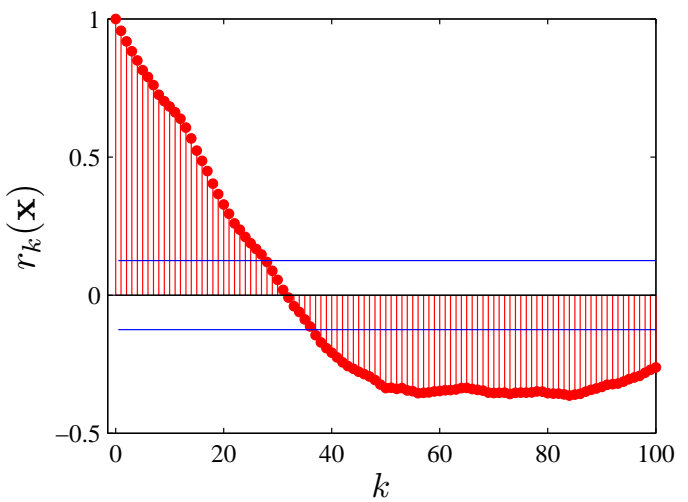

(b)

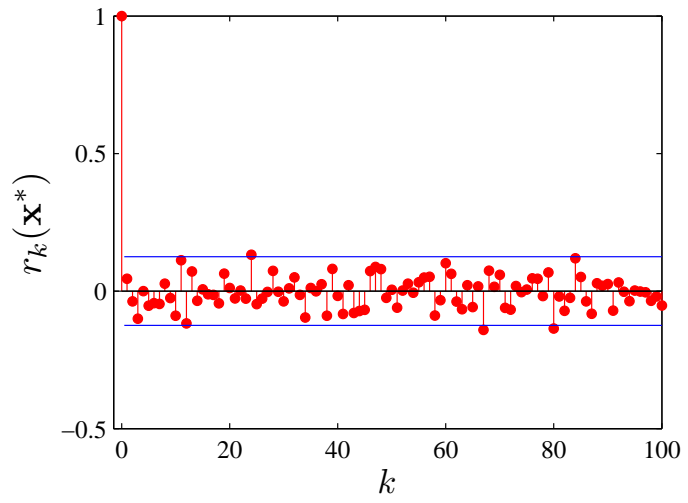

(d)

Figura 2.5: (a) Série temporal tomada de um modelo autoregressivo de ordem 1 sendo $A(z)=1-0,95 z^{-1}$ e $N=256$; (b) Correlograma de $x(n)$ para $k=100$; (c) Reamostragem $x^{*}(n)$ de $x(n)$; (d) Correlograma de $x^{*}(n)$.

métodos sobre séries temporais provenientes de exames de ressonância magnética funcional (fMRI).

\subsection{Outros Métodos de Reamostragem de Séries Correlacionadas}

Além dos métodos que fazem o uso de ferramentas de pre-whitening, existem aqueles que são feitos no domínio do tempo como é o caso da reamostragem por blocos, conhecido como moving block bootstrap (MBB), em que a reamostragem é feita em blocos $B_{i}$ de comprimento $l$ do sinal e cujo diagrama é mostrado na figura 2.6.

A grande dificuldade deste método reside em se determinar $l$ conveniente para a aplicação. Como variações do MBB, é possível citar o nonoverlapping block bootstrap (NBB) em que os blocos são escolhidos de tal forma que não haja sobreposição entre os mesmos, o circular block bootstrap (CBB) em que as amostras são colocadas em forma circular e salvam os casos em que o comprimento $N$ da série não é múltiplo do comprimento $l$ dos blocos e o stationary block 


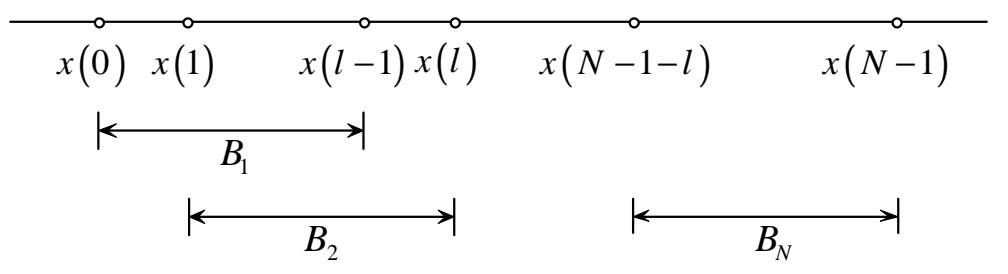

Figura 2.6: Diagrama do moving block bootstrap em que são escolhidos trechos de comprimento $l$ do sinal para serem reamostrados de acordo com os procedimentos 3 e 4.

bootstrap (SBB) em que o comprimento $l$ dos blocos é variável. Todos estes métodos são amplamente discutidos em [21].

Além das citadas acima, uma outra alternativa é a reamostragem baseada em resíduos de modelos pré-ajustados aos dados, discutida em [3, 22]. 


\section{Decomposição Wavelet}

\subsection{Motivação}

A transformada wavelet discreta (DWT) de tempo discreto é uma ferramenta poderosa nas questões que tangem os conceitos de reamostragem de séries temporais pois possui, sob certas condições, os coeficientes descorrelacionados $[5,6,7,8,9]$, o que acaba motivando sua aplicação como ferramenta de prewhitening.

Nesta seção apresentamos alguns aspectos que dizem respeito à teoria envolvida na decomposição wavelet, como base para entendimento dos procedimentos de reamostragem apresentados subsequentemente.

\subsection{Transformada Wavelet Discreta de Tempo Discreto}

A transformada wavelet discreta decompõe um sinal $x(n)$ em coeficientes associados a diferentes escalas e tempos, ou seja, é uma decomposição do tipo 'tempo/escala' cuja maneira mais elegante encontrada na literatura para introduzila é por meio de matrizes ortogonais [11]. Sendo assim, ela tranforma um sinal

$$
\mathbf{x}=[x(0) \cdots x(N-1)]^{T}
$$

com $N=2^{J}$, em um vetor $\mathbf{W}$, também de comprimento $N$, contendo seus coeficientes por meio de uma matriz ortogonal $\mathcal{W}_{N \times N}$ através da equação de análise,

$$
\mathbf{W}=\mathcal{W} \mathbf{x}
$$

Além disto, o fato de $\mathcal{W}$ ser ortogonal garante que o sinal x possa ser recuperado pela equação de síntese,

$$
\mathbf{x}=\mathcal{W}^{T} \mathbf{W} .
$$


Considerando que $\mathbf{x}$ seja decomposto em $J_{0} \leq J$ níveis, o vetor de coeficientes transformados

$$
\mathbf{W}=\left[\begin{array}{c}
W(0) \\
\vdots \\
W(N-1)
\end{array}\right]
$$

pode ser particionado como [11]

$$
\mathbf{W}=\left[\begin{array}{c}
\mathbf{W}_{1} \\
\vdots \\
\mathbf{W}_{J_{0}} \\
\mathbf{V}_{J_{0}}
\end{array}\right]
$$

com,

$$
\mathbf{W}_{j}=\left[\begin{array}{lll}
W\left(\frac{2^{j-1}-1}{2^{j-1}} N\right) & \cdots & W\left(\frac{2^{j}-1}{2^{j}} N-1\right)
\end{array}\right]^{T}
$$

$\mathrm{e}$

$$
\mathbf{V}_{J_{0}}=\left[\begin{array}{llll}
W\left(\frac{2^{J_{0}}-1}{2^{J_{0}}} N\right) & \cdots & W(N-1)
\end{array}\right]^{T},
$$

para $1 \leq j \leq J_{0}$

Foi utilizando essa interpretação que Mallat [4] introduziu o algoritmo piramidal, ilustrado nas figuras 3.1 e 3.2 para $J_{0}=3$. Esse algoritmo está ligado aos conceitos de bancos de filtros $[23,24]$ e sua conexão com a representação matricial [11] é feita pela matriz $\mathcal{W}$ que depende explicitamente dos coeficientes $\{h(n): n=0, \cdots, L-1\}, L$ par, do filtro $H(z)$ que por sua vez dependem da wavelet adotada.

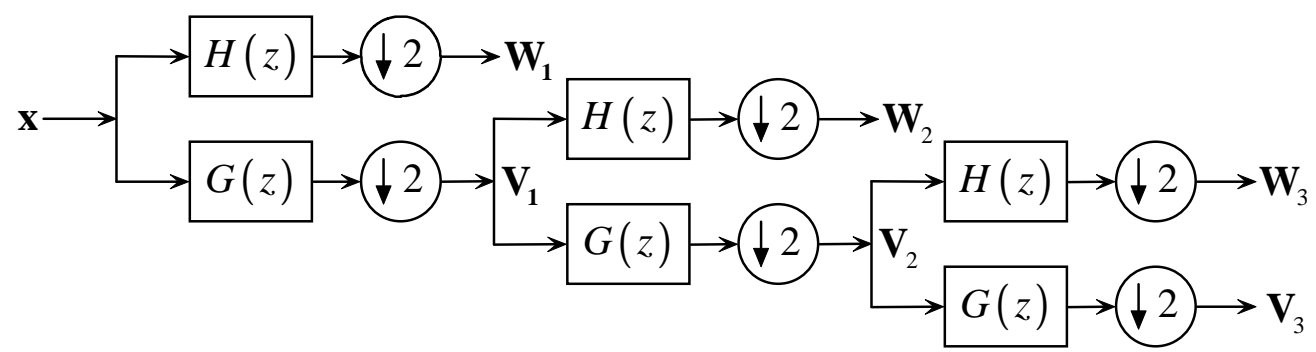

Figura 3.1: DWT - Decomposição para $J_{0}=3$.

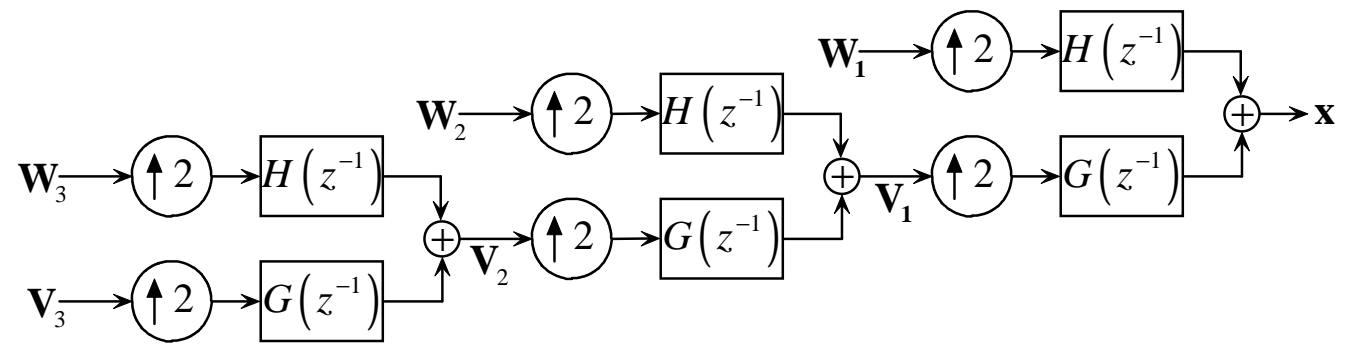

Figura 3.2: DWT - Reconstrução para $J_{0}=3$. 
Este banco de filtros é conhecido na literatura como banco QMF ( Quadrature Mirror Filters), devido aos filtros $H(z)$ e $G(z)$ estarem relacionados por

$$
G(z)=z^{-(L-1)} H\left(-z^{-1}\right)
$$

Sendo assim, dados os coeficientes $\{h(n): n=0, \cdots, L-1\}$ do filtro $H(z)$, é possível determinar os coeficientes $\{g(n): n=0, \cdots, L-1\}$ do filtro $G(z)$ por meio da seguinte relação (transformada inversa da eq. 3.7 ),

$$
g(n)=(-1)^{n+1} h(L-1-n)
$$

Uma importante observação a ser feita é com relação a causalidade dos filtros, ou seja, conforme pode-se ver, os filtros $H\left(z^{-1}\right)$ e $G\left(z^{-1}\right)$ são as versões anticausais de $H(z)$ e $G(z)$, respectivamente, o que garante a reconstrução perfeita sem atraso, demonstrada no anexo A. Ocorre que, para a implementação do algoritmo piramidal através de DSP's, o que não é o caso aqui, é necessário que os filtros sejam todos causais, implicando numa reconstrução perfeita com atraso.

Os coeficientes W da DWT particionados de acordo com a equação 3.4 são relacionados com deteminadas faixas de frequência do espectro conforme podemos ver na figura 3.3 .

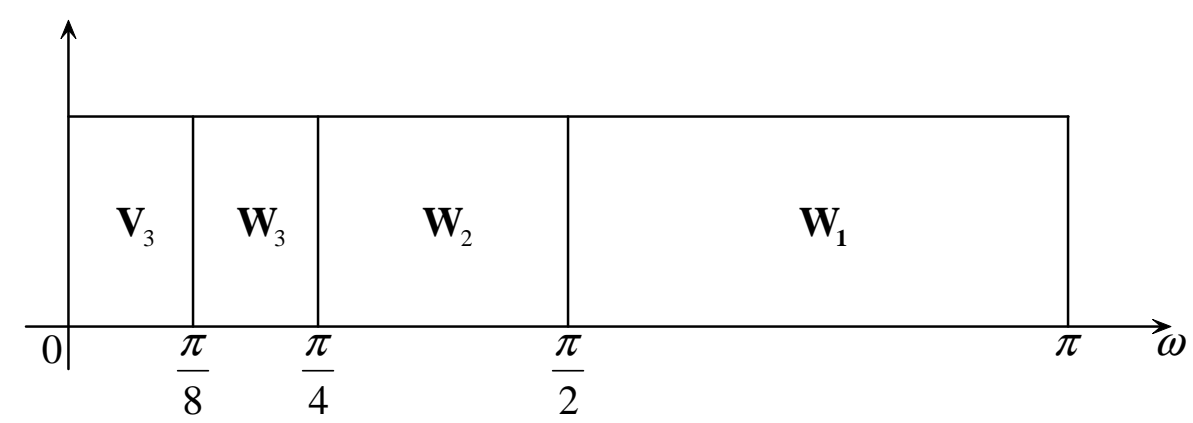

Figura 3.3: DWT - Relação entre os coeficientes e suas respectivas bandas espectrais.

Obviamente que o corte entre as bandas não é abrupto conforme esboça a figura 3.3, que deve-se ao fato de $H(z)$ e $G(z)$ não serem ideais.

Outra característica importante da DWT, que deve ser levada em consideração nos algoritmos de reamostragem, é a decomposição da energia do sinal entre as escalas, ou seja,

$$
\|\mathbf{x}\|^{2}=\sum_{j=1}^{J_{0}}\left\|\mathbf{W}_{j}\right\|^{2}+\left\|\mathbf{V}_{J_{0}}\right\|^{2} .
$$




\subsection{Transformada Wavelet Packet Discreta de Tempo Discreto}

A DWPT de tempo discreto é um tipo de transformação 'tempo/frequência' [11], assim como a short-time Fourier transform (STFT), e a grande motivação para sua aplicação aqui está no fato de ser possível decompôr a banda $[\pi / 2, \pi]$, deixada de lado pela DWT.

Sua descrição por meio de matrizes ortogonais é semelhante a da DWT, isto é, as mesmas equações de análise e síntese são preservadas, mudando simplesmente a forma como a matriz $\mathcal{W}$ é construída [11].

Esta nova construção de $\mathcal{W}$ permite um particionamento diferente do vetor de coeficientes $\mathbf{W}$ da forma,

$$
\mathbf{W}=\left[\begin{array}{c}
\mathbf{W}_{J_{0}, 0} \\
\vdots \\
\mathbf{W}_{J_{0}, 2^{J_{0}-1}}
\end{array}\right]
$$

com,

$$
\mathbf{W}_{J_{0}, k}=\left[\begin{array}{lll}
W\left(\frac{k}{2^{J_{0}}} N\right) & \cdots & W\left(\frac{k+1}{2^{J_{0}}} N-1\right)
\end{array}\right]^{T},
$$

para $0 \leq k \leq 2^{J_{0}}-1$.

Nesta decomposição a energia também é conservada, como no caso anterior, ou seja,

$$
\|\mathbf{x}\|^{2}=\sum_{j=1}^{J_{0}} \sum_{k=0}^{2^{J_{0}-1}}\left\|\mathbf{W}_{j, k}\right\|^{2}
$$

Os algoritmos piramidais de decomposição e reconstrução da DWPT para o caso $J_{0}=3$ são mostrados, respectivamente, nas figuras 3.4 e 3.5 , sendo que o banco de filtros utilizado é o mesmo usado na DWT.

Uma forma mais elegante de visualizar a decomposição DWPT está simbolizada na figura 3.7 para $J_{0}=3$, onde pode-se ver que a reconstrução pode ser feita por caminhos alternativos, ou seja, ao invés de utilizar todos os subvetores de nível $j=3$, é possível reconstruir o sinal, por exemplo, utilizando os subvetores hachurados ilustrados na figura. Na referência [11] o autor estabelece um critério baseado em menor entropia para determinar o caminho para a reconstrução. Aqui são considerados todos os subvetores de mesmo nível (Capítulo 4).

O relacionamento dos coeficientes da DWPT com as respectivas bandas pode ser apreciado na figura 3.6. 


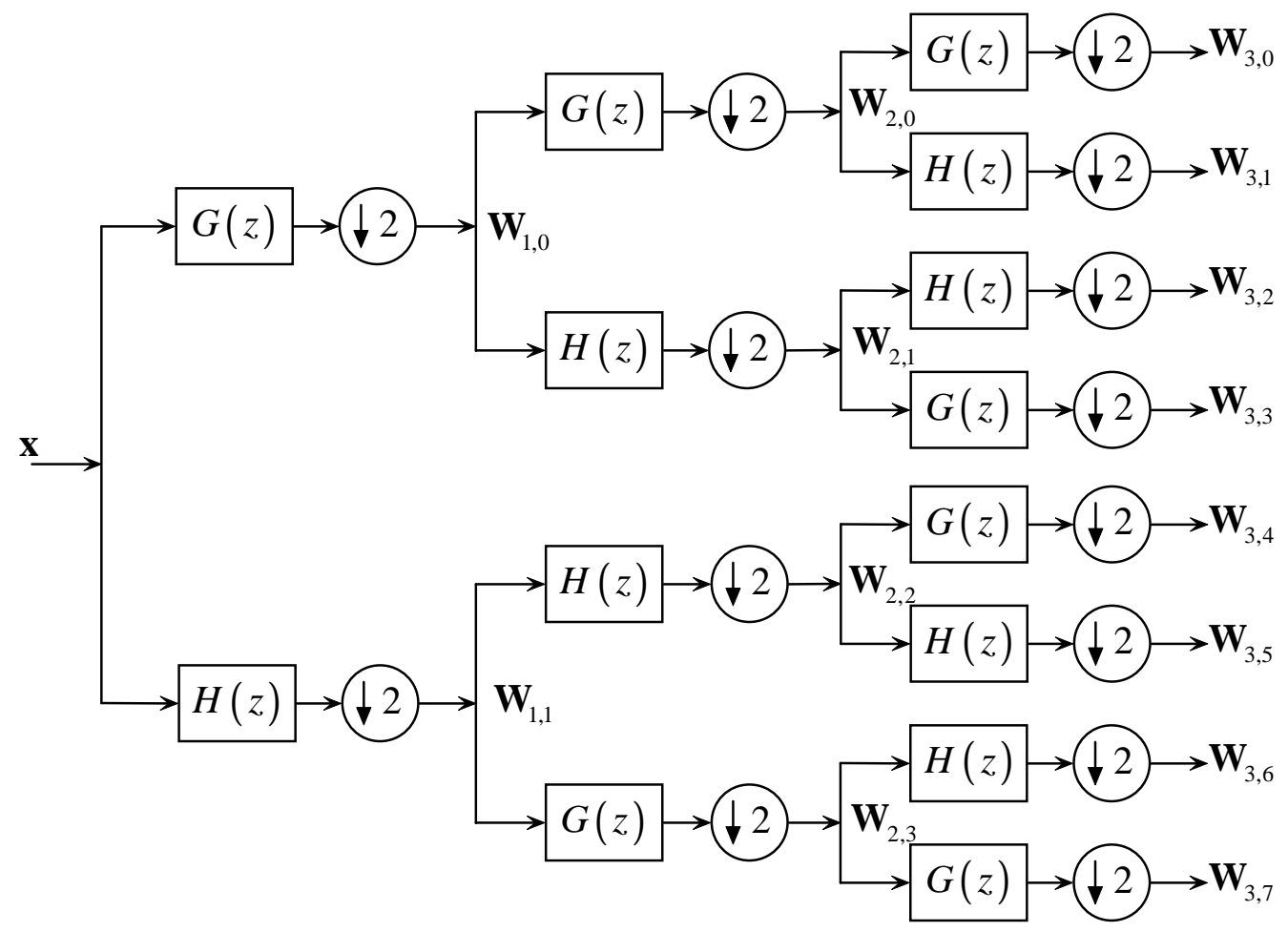

Figura 3.4: DWPT - Decomposição para $J_{0}=3$.

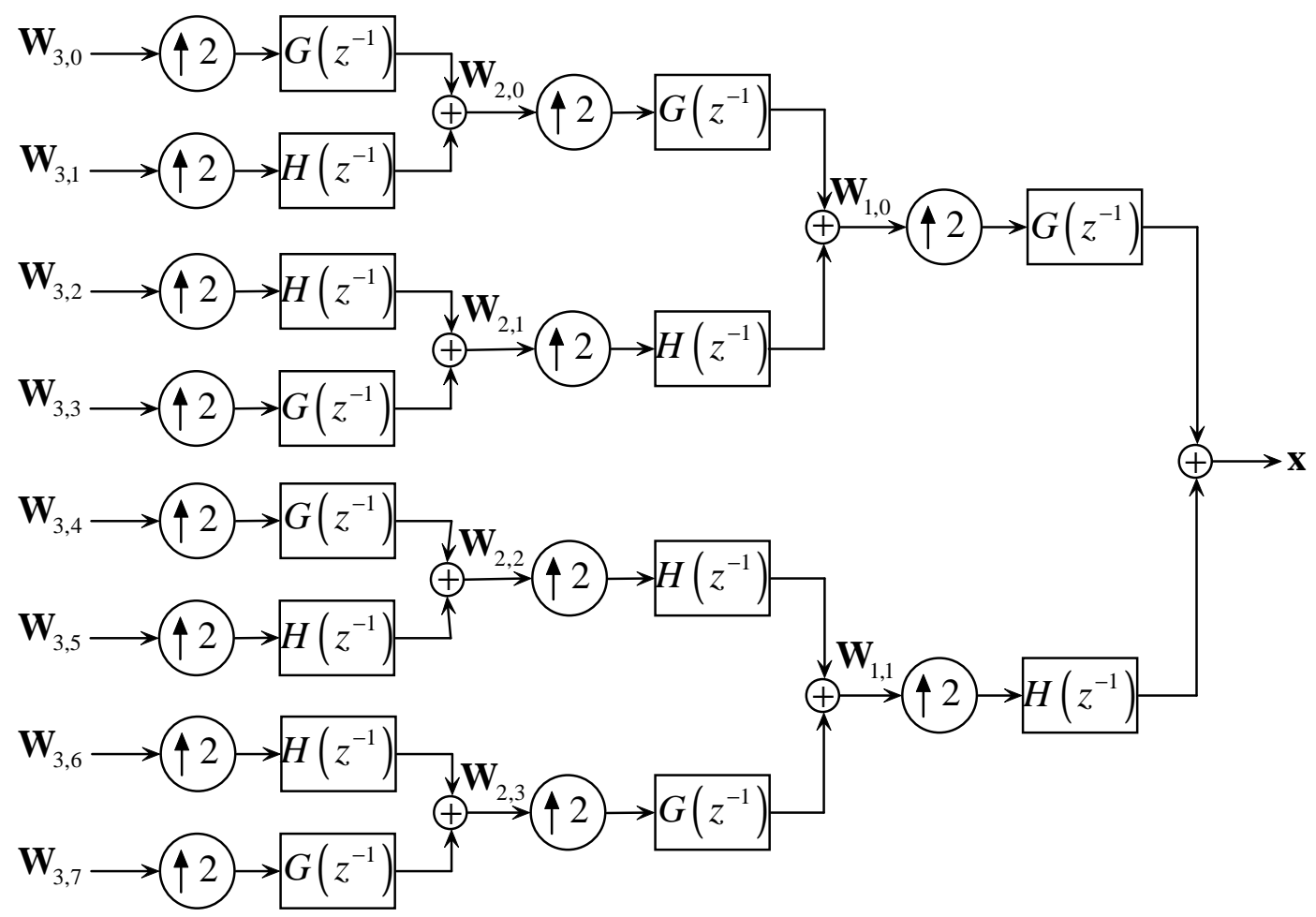

Figura 3.5: DWPT - Reconstrução para $J_{0}=3$. 


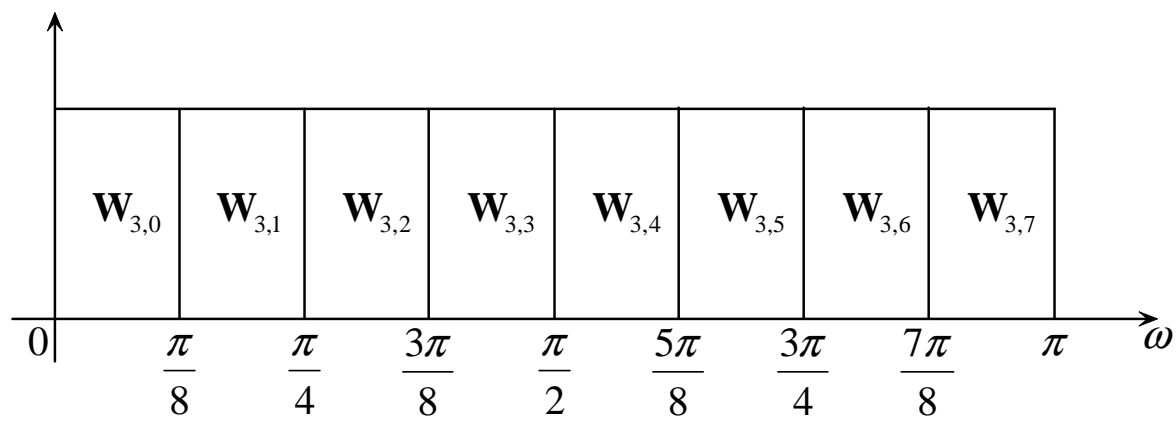

Figura 3.6: DWPT - Relação entre os coeficientes e suas respectivas bandas espectrais.

\begin{tabular}{|c|c|c|c|c|c|c|c|c|}
\hline$j=0$ & \multicolumn{8}{|c|}{$\mathbf{W}_{0,0}=\mathbf{x}$} \\
\hline$j=1$ & \multicolumn{4}{|c|}{$\mathbf{W}_{1,0}$} & \multicolumn{4}{|c|}{$\mathbf{W}_{1,1}$} \\
\hline$j=2$ & \multicolumn{2}{|c|}{$\mathbf{W}_{2,0}$} & \multicolumn{2}{|c|}{$\mathbf{W}_{2,1}$} & \multicolumn{2}{|c|}{$\mathbf{W}_{2,2}$} & \multicolumn{2}{|c|}{$\mathbf{W}_{2,3}$} \\
\hline$j=3$ & $\mathbf{W}_{3,0}$ & $\mathbf{W}_{3,1}$ & $\mathbf{W}_{3,2}$ & $\mathbf{W}_{3,3}$ & $\mathbf{W}_{3,4}$ & $\mathbf{W}_{3,5}$ & $\mathbf{W}_{3,6}$ & $\mathbf{W}_{3,7}$ \\
\hline & ) & $\pi$ & $\tau$ & $\frac{\pi}{\pi}$ & $\pi$ & $\pi$ & $\frac{1}{\pi}$ & $\pi$ \\
\hline & & 8 & 4 & 8 & 2 & $\overline{8}$ & 4 & \\
\hline
\end{tabular}

Figura 3.7: Caminho alternativo para reconstrução DWPT.

As rotinas utilizadas neste estudo para decomposição e reconstrução DWT e DWPT, implementadas em MATLAB, são apresentadas no anexo C e exemplos numéricos de decomposição, assim como alguns aspectos sobre a construção da matriz $\mathcal{W}$ são mostrados no anexo $\mathrm{B}$. 


\section{Parte II}

Análise Experimental 


\section{Reamostragem Baseada em Wavelets}

Neste capítulo são apresentados dois métodos de reamostragem baseados em wavelets, conhecidos na literatura como wavestrap e wavestrapping, e que fazem uso da DWT e DWPT, respectivamente. Suas aplicações são na estimação da densidade espectral de potência de um processo AR.

\subsection{Procedimentos de Wavestrap e Wavestrap- ping}

Na reamostragem baseada em wavelets (procedimentos 5 e 6), cujo diagrama é apresentado na figura 4.1, uma série temporal observada $x(n)$ é primeiramente decomposta, fazendo-se em seguida a reamostragem dos coeficientes através dos procedimentos 3 ou 4 seguido ao cálculo da transformada inversa, gerando a versão reamostrada $x^{*}(n)$ da série original. Este método de reamostragem é válido, pois os coeficientes wavelet, sob certas condições particulares, são descorrelacionados.

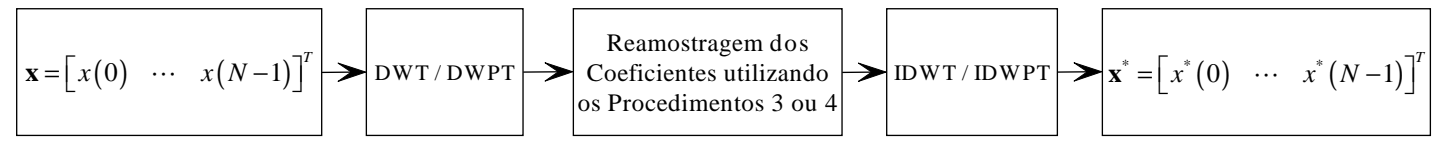

Figura 4.1: Diagrama da reamostragem baseada em wavelets.

Algumas destas condições ainda vêm sendo estudadas. Dentre elas, são investigadas aqui:

1. Tipo e ordem do filtro wavelet empregado na decomposição;

2. Número de níveis utilizados na decomposição;

3. Presença de picos na densidade espectral de potência das séries ${ }^{1}$;

\footnotetext{
${ }^{1}$ Caractarizado pela presença de polos próximos à circunferência unitária.
} 
Procedimento 5 Wavestrap: Densidade Espectral de Potência

Entrada: $\mathbf{x}=[x(0) \cdots x(N-1)]^{T}$ e $J_{0}$

1. Escolha a wavelet desejada assim como sua ordem e monte a matriz $\mathcal{W}$ [11].

2. Determine a DWT de $\mathbf{x}$ através da equação de análise,

$$
\mathbf{W}=\left[\begin{array}{c}
\mathbf{W}_{1} \\
\vdots \\
\mathbf{W}_{J_{0}} \\
\mathbf{V}_{J_{0}}
\end{array}\right]=\mathcal{W} \mathbf{x}
$$

3. Reamostre sem reposição os coeficientes dos subvetores $\mathbf{W}_{1}, \cdots \mathbf{W}_{J_{0}}, \mathbf{V}_{J_{0}}$, e obtenha

$$
\mathbf{W}^{*}=\left[\begin{array}{c}
\mathbf{W}_{1}^{*} \\
\vdots \\
\mathbf{W}_{J_{0}}^{*} \\
\mathbf{V}_{J_{0}}^{*}
\end{array}\right] .
$$

4. Calcule o sinal reamostrado através da equação de síntese da DWT,

$$
\mathbf{x}^{*}=\mathcal{W}^{T} \mathbf{W}^{*}
$$

5. Determine uma estimativa $\hat{S}_{x}^{*}\left(e^{j \omega}\right)$ para a densidade espectral de potência $S_{x}\left(e^{j \omega}\right)$ de $x(n)$ utilizando a versão reamostrada $x^{*}(n)$ e um estimador apropriado.

Saída: $\hat{S}_{x}^{*}\left(e^{j \omega}\right)$

Procedimento 6 Wavestrapping: Densidade Espectral de Potência

Entrada: $\mathbf{x}=[x(0) \cdots x(N-1)]^{T}$ e $J_{0}$

1. Escolha a wavelet desejada assim como sua ordem e monte a matriz $\mathcal{W}[11]$.

2. Determine a DWPT de $\mathbf{x}$ através da equação de análise,

$$
\mathbf{W}=\left[\begin{array}{c}
\mathbf{W}_{J_{0}, 0} \\
\vdots \\
\mathbf{W}_{J_{0}, 2^{J_{0}}-1}
\end{array}\right]=\mathcal{W} \mathbf{x} .
$$

3. Reamostre sem reposição os coeficientes dos subvetores $\mathbf{W}_{J_{0}, 0} \cdots \mathbf{W}_{J_{0}, 2^{J_{0}-1}}$ e obtenha

$$
\mathbf{W}^{*}=\left[\begin{array}{c}
\mathbf{W}_{J_{0}, 0}^{*} \\
\vdots \\
\mathbf{W}_{J_{0}, 2^{J_{0}-1}}^{*}
\end{array}\right] .
$$

4. Calcule o sinal reamostrado através da equação de síntese da DWPT,

$$
\mathbf{x}^{*}=\mathcal{W}^{T} \mathbf{W}^{*}
$$

5. Determine uma estimativa $\hat{S}_{x}^{*}\left(e^{j \omega}\right)$ para a densidade espectral de potência $S_{x}\left(e^{j \omega}\right)$ de $x(n)$ utilizando a versão reamostrada $x^{*}(n)$ e um estimador apropriado.

Saída: $\hat{S}_{x}^{*}\left(e^{j \omega}\right)$ 
Dentre as condições listadas a que provavelmente têm sido mais estudada é a terceira, ou seja, é de interesse saber como os procedimentos 5 e 6 se comportam quando no espectro existem picos. Por isso, usam-se aqui as séries autoregressivas, geradas a partir de sistemas que possuem pólos próximos à circunferência unitária.

Neste texto, na investigação da primeira condição são utilizadas as wavelets de Daubechies devido aos filtros não apresentarem tanto vazamento. Outra opção interessante de wavelets são as conhecidas como least asymmetric ou symmlets $[25,11]$, que são uma variação das anteriores, também introduzidas por Ingrid Daubechies [25].

Uma nota importante com relação aos procedimentos 5 e 6 é a escolha entre as observações ser feita sem reposição. Isto precisa ser respeitado devido à necessidade da manutenção da energia da série.

\subsection{Cenários de Estudo}

Aqui são consideradas séries temporais autoregressivas $x_{i}(n) \operatorname{com} i=1, \cdots, 3$ geradas conforme a figura 4.2 sendo

$$
S_{i}(z)=\frac{1}{A_{i}(z)}
$$

tal que,

$$
\begin{aligned}
& \qquad A_{1}(z)=1+0,4 z^{-1}+0,2 z^{-2}+0,1 z^{-3}+0,1 z^{-4}+0,1 z^{-5} \\
& \qquad A_{2}(z)=1+0,950 z^{-2} \\
& A_{3}(z)=1-0,6119 z^{-1}+0,3744 z^{-2}+0,5705 z^{-3}+0,3668 z^{-4}-0,5877 z^{-5}+0,9414 z^{-4} \\
& \text { cujas respostas em frequência normalizada estão ilustradas na figura 4.3. Neste } \\
& \text { texto todas as estimativas espectrais }\left|S_{i}\left(e^{j \omega}\right)\right|^{2} \text { são normalizadas em potência, e } \\
& \text { denotadas simplesmente por } S_{i_{N}}\left(e^{j \omega}\right) \text {, devido à ênfase aqui estar na posição dos } \\
& \text { picos. }
\end{aligned}
$$

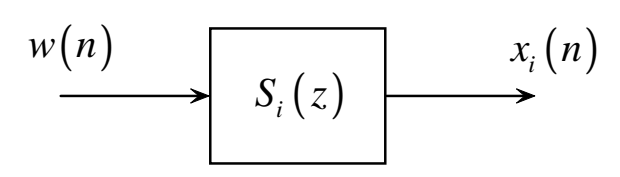

Figura 4.2: Geração de uma série temporal por um filtro AR.

É interessante notar que $x_{2}(n)$ e $x_{3}(n)$ foram escolhidas de forma que em suas representações espectrais apareçam picos acentuados em regiões estratégicas, ao passo que série $x_{1}(n)$, emprestada de [5], não possui tais picos. 


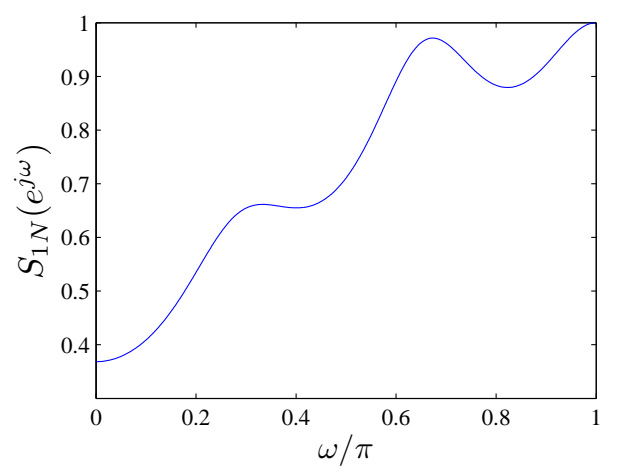

(a)

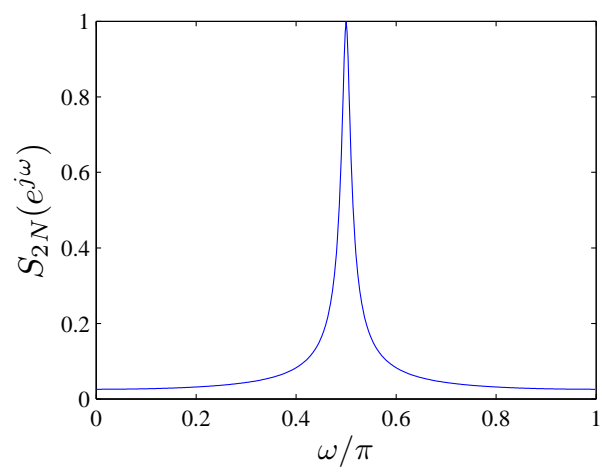

(b)

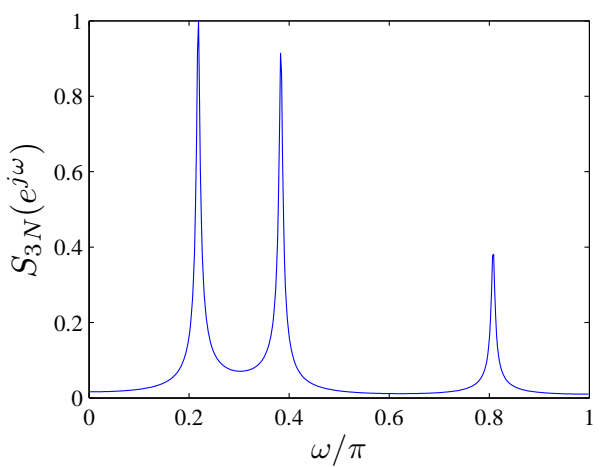

(c)

Figura 4.3: Respostas em frequência normalizadas dos filtros AR considerados no estudo.

Estes picos estreitos e acentuados prejudicam os resultados apresentados pela reamostragem wavestrap, pois as observações de tais sinais não são descorrelacionadas após a transformação. Isto, normalmente é o que justifica a aplicação do método de wavestrapping.

\subsubsection{Considerações sobre a Ordem do Filtro Wavelet}

Nesta seção são estudados os métodos quando o número de níveis de decomposição é mantido constante em $J_{0}=4$ e a ordem $L$ dos filtros é variada. A ordem do filtro wavelet empregado é uma etapa importante nos processos de reamostragem que os utilizam, pois conforme a ordem varia, as bandas dos filtros utilizados na decomposição variam, ou melhor, quanto maior for a ordem, mais estreitos são os filtros, menos vazamento há entre as bandas e, com isso, as porções de espectro do sinal comuns a duas bandas adjacentes são menores (figura 4.4).

Por outro lado, um certo cuidado deve ser tomado na escolha da ordem dos filtros pois, se forem escolhidas ordens muito elevadas, o esforço computacional aumenta consideravelmente e em alguns casos o resultado da reamostragem não melhora. Sendo assim, para ser estabelecida a ordem do filtro wavelet, e desta forma descorrelacionar a série, é necessário que seja feita uma avaliação do espec- 


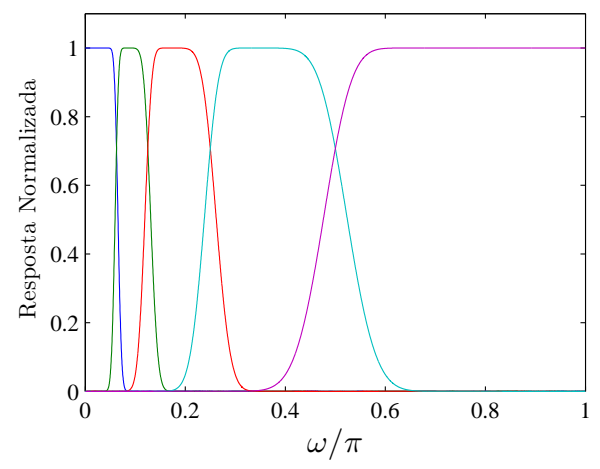

(a)

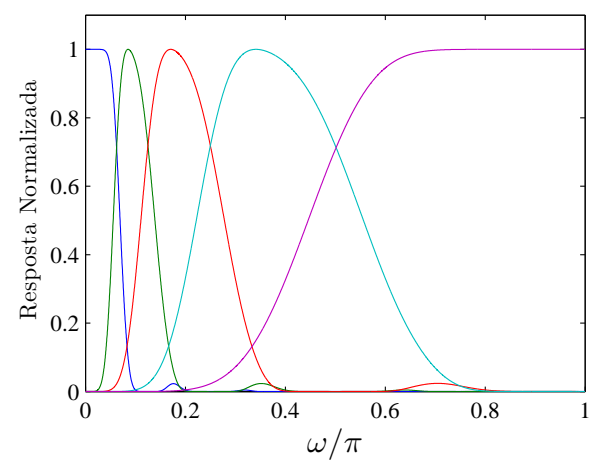

(b)

Figura 4.4: Resposta em frequência normalizada dos filtros de decomposição DWT em 4 níveis utilizando as wavelets de Daubechies de ordens: (a) 80; (b) 16.

tro de modo que, os picos existentes não pertençam a uma única banda, ou seja, para que a série seja descorrelacionada, a potência dos picos deve ser distribuída entre bandas adjacentes sem que haja sobreposição (situação praticamente impossível de ser alcançada devido aos filtros não serem ideais).

Como primeiro exemplo considera-se a série $x_{1}(n)$, com $N=1024$ (figura 4.5(a)) assim como uma estimativa para sua densidade espectral de potência utilizando o método de Burg [26, 27, 28] (figura 4.5(b)).

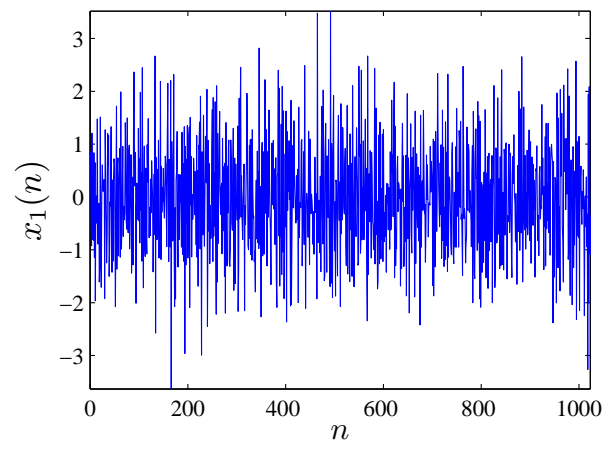

(a)

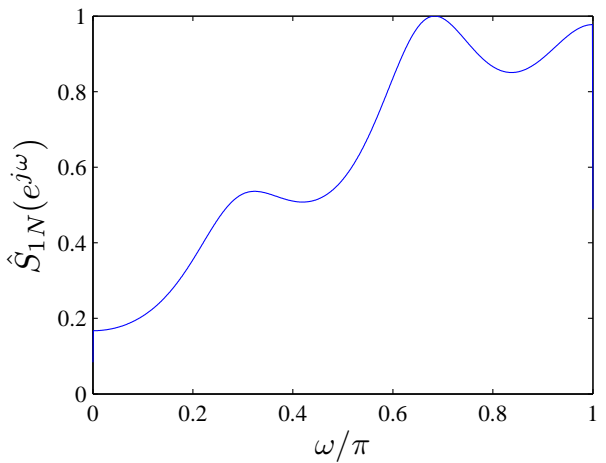

(b)

Figura 4.5: (a) Primeira série temporal $\left(x_{1}(n)\right)$; (b) Seu espectro de potência normalizado $\left(\hat{S}_{1 N}\left(e^{j \omega}\right)\right)$.

Nas figuras 4.6 e 4.7 são apresentadas, respectivamente, as autocorrelações dos coeficientes na decomposição de $x_{1}(n)$ em 4 níveis utilizando a DWT e DWPT com filtros Daubechies de ordem 8. Comparando estas figuras é possível notar que todos os coeficientes dentro de um mesmo subvetor, de ambas as transformadas, são aproximadamente descorrelacionados. Isto garante que os procedimentos $5 \mathrm{e}$ 6 apresentem resultados satisfatórios.

Esta eficácia também pode ser justificada no domínio da frequência. Na figura 4.8(a) é apresentada a resposta em frequência $S_{1 N}\left(e^{j \omega}\right)$ sobreposta às respostas dos filtros wavelet utilizados na decomposição DWT, onde se nota que 

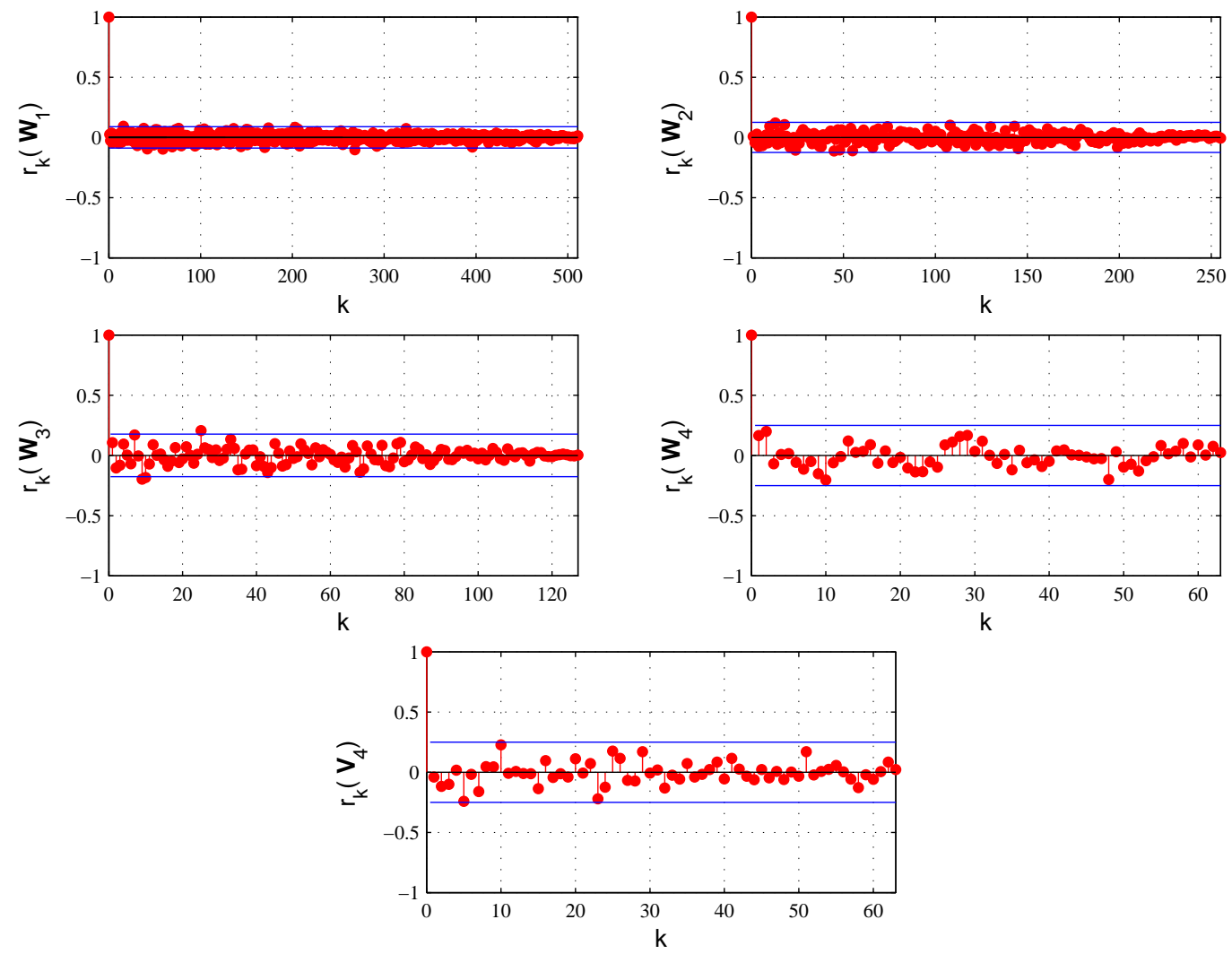

Figura 4.6: Autocorrelações dos coeficientes da decomposição DWT de $x_{1}(n)$ em 4 níveis utilizando filtros Daubechies de ordem 8.
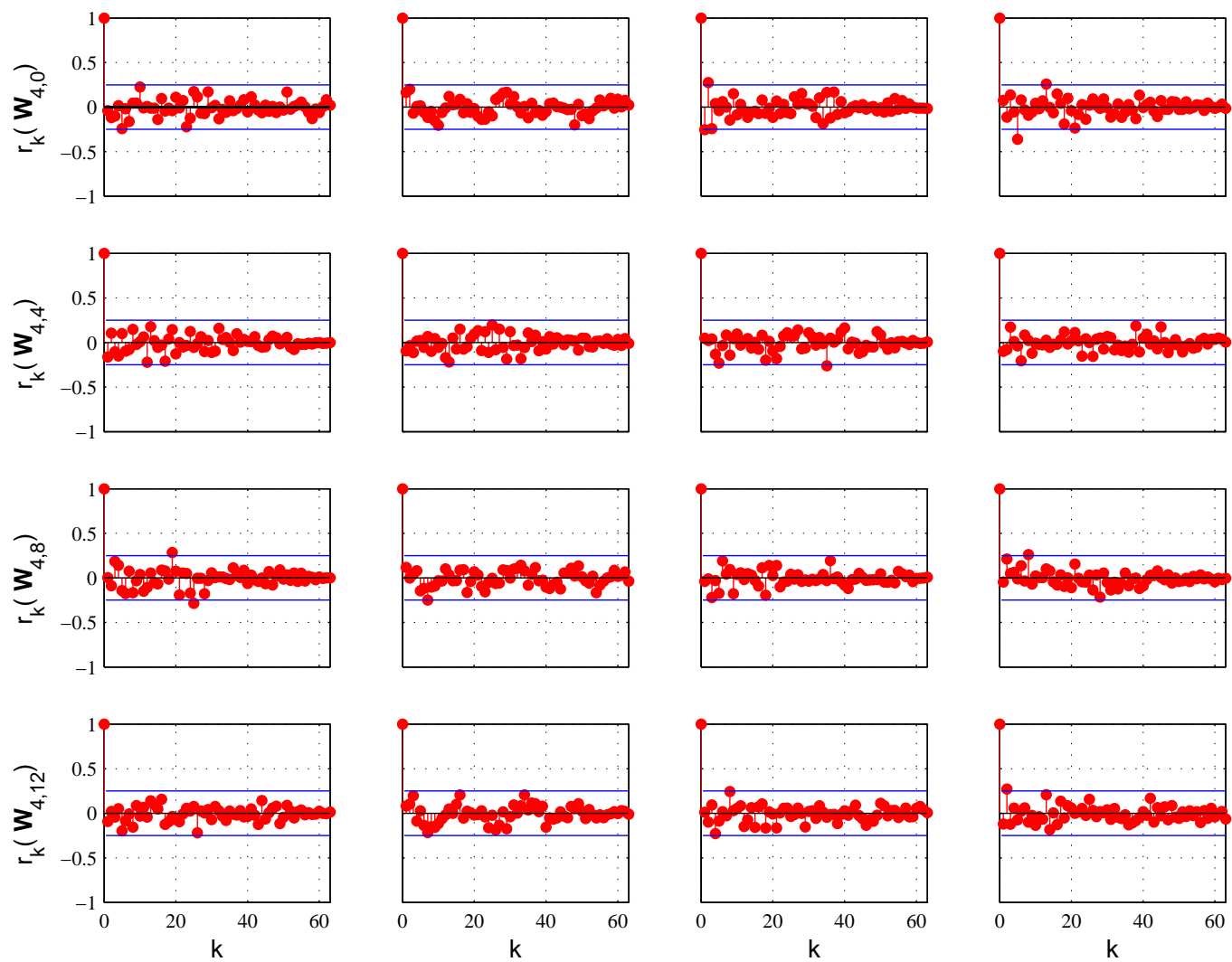

Figura 4.7: Autocorrelações dos coeficientes da decomposição DWPT de $x_{1}(n)$ em 4 níveis utilizando filtros Daubechies de ordem 8. 


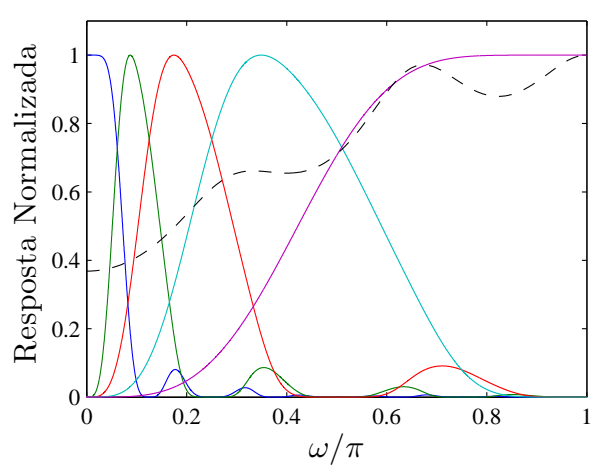

(a)

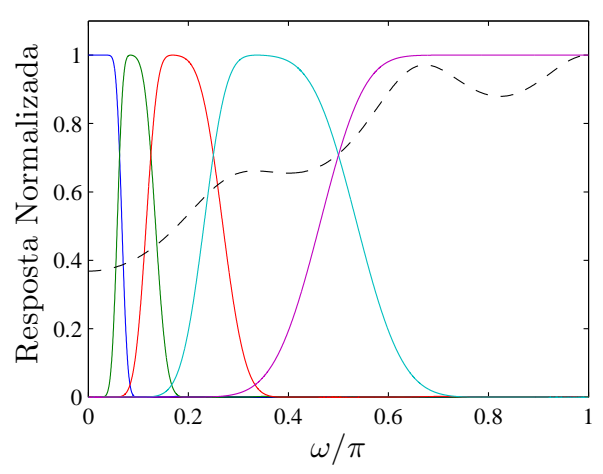

(b)

Figura 4.8: Resposta $S_{1 N}\left(e^{j \omega}\right)$ sobreposta à resposta dos filtros Daubechies, na decomposição DWT de 4 níveis, de ordem (a) 8; (b) 32.

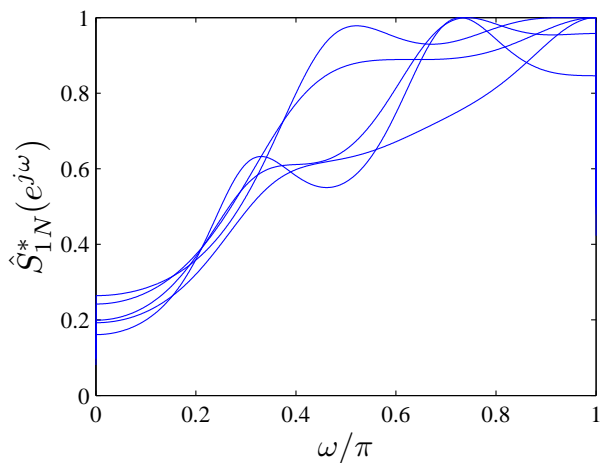

(a)

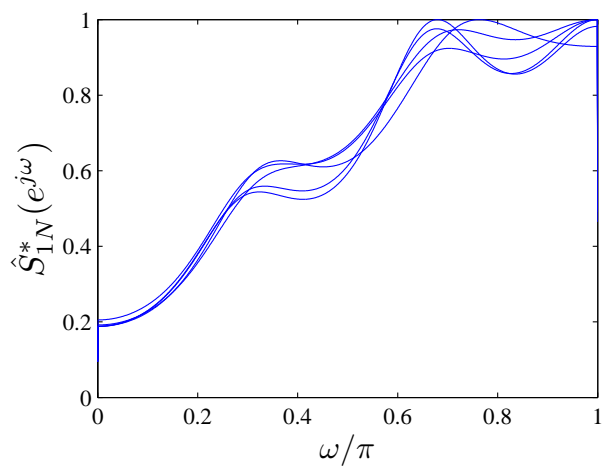

(b)

Figura 4.9: Estimativas de $S_{1 N}\left(e^{j \omega}\right)$ utilizando decomposições em 4 níveis com filtros Daubechies de ordem 8 dadas pelos procedimentos de (a) wavestrap; (b) wavestrapping.

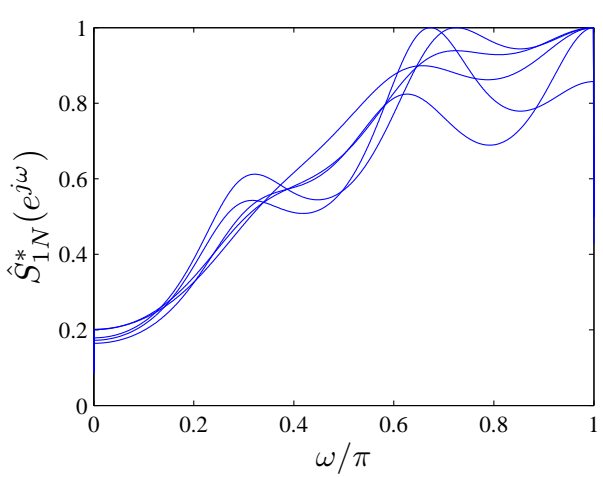

(a)

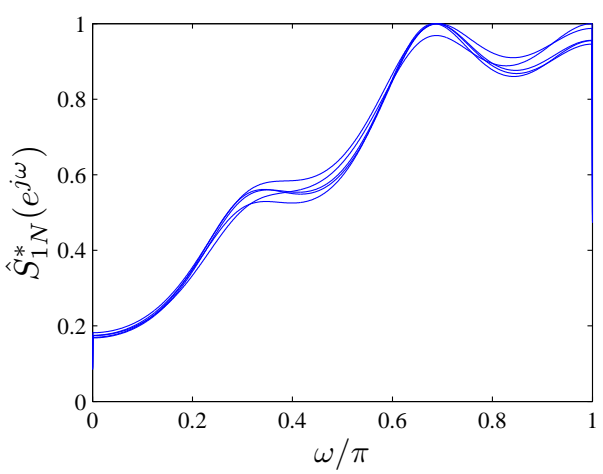

(b)

Figura 4.10: Estimativas de $S_{1 N}\left(e^{j \omega}\right)$ utilizando decomposições em 4 níveis com filtros Daubechies de ordem 32 dadas pelos procedimentos de (a) wavestrap; (b) wavestrapping. 
praticamente não há porções espectrais comuns a duas bandas de decomposição, garantindo o resultado.

Aplicando 5 vezes os respectivos procedimentos sobre $x_{1}(n)$, são obtidas 5 séries reamostradas pela DWT e 5 pela DWPT cujas estimativas espectrais (figura 4.9) revelam certa superioridade do procedimento de wavestrapping sobre o wavestrap. Isto ocorre devido a DWPT decompôr a banda $\pi / 2 \leq \omega \leq \pi$, deixada de lado pela DWT.

Em contrapartida, o resultado apresentado pelo wavestrap (figura 4.9(a)) não deve ser desconsiderado, pois além de não apresentar viés (somente variabilidade maior), este método é mais eficiente computacionalmente.

Aumentando a ordem dos filtros wavelet, por exemplo, de 8 para 32, e repetindo o processo, tem-se uma melhoria pouco significativa nos resultados (figura 4.10), obtida devido ao vazamento entre as bandas ser menor (figura 4.8(b)).

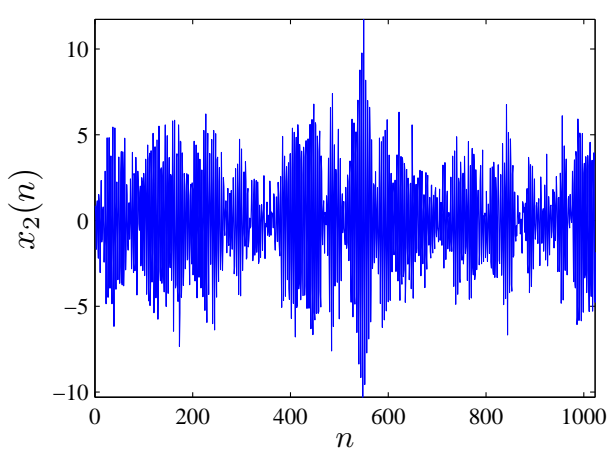

(a)

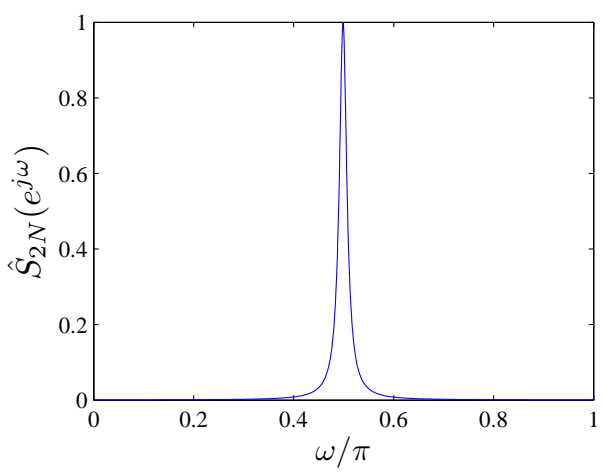

(b)

Figura 4.11: (a) Segunda série temporal $\left(x_{2}(n)\right)$; (b) Seu espectro de potência normalizado $\left(\hat{S}_{2 N}\left(e^{j \omega}\right)\right)$.

Calculando agora as autocorrelações dos coeficientes da decomposição de $x_{2}(n)$ (figura 4.11) em 4 níveis pelas DWT e DWPT com filtros Daubechies de ordem 16 (figuras 4.12 e 4.13), é possível ver que os coeficientes presentes nos subvetores $\mathbf{W}_{1}$ e $\mathbf{W}_{2}$ da DWT são fortemente correlacionados, ao passo que os coeficientes presentes nos subvetores da DWPT são todos praticamente descorrelacionados, com exceção de $\mathbf{W}_{4,7}$ e $\mathbf{W}_{4,8}$ que apresentam uma pequena correlação, aqui desprezada.

Aplicando, por exemplo, 5 vezes os procedimentos 5 e 6 e determinando as estimativas espectrais pelo método de Burg (figura 4.15) é possível ver que o wavestrap apresenta viés estatístico, ao passo que o wavestrapping mostra-se bem mais eficaz.

Isto pode ser justificado por meio da figura 4.14(a) onde são mostradas as 

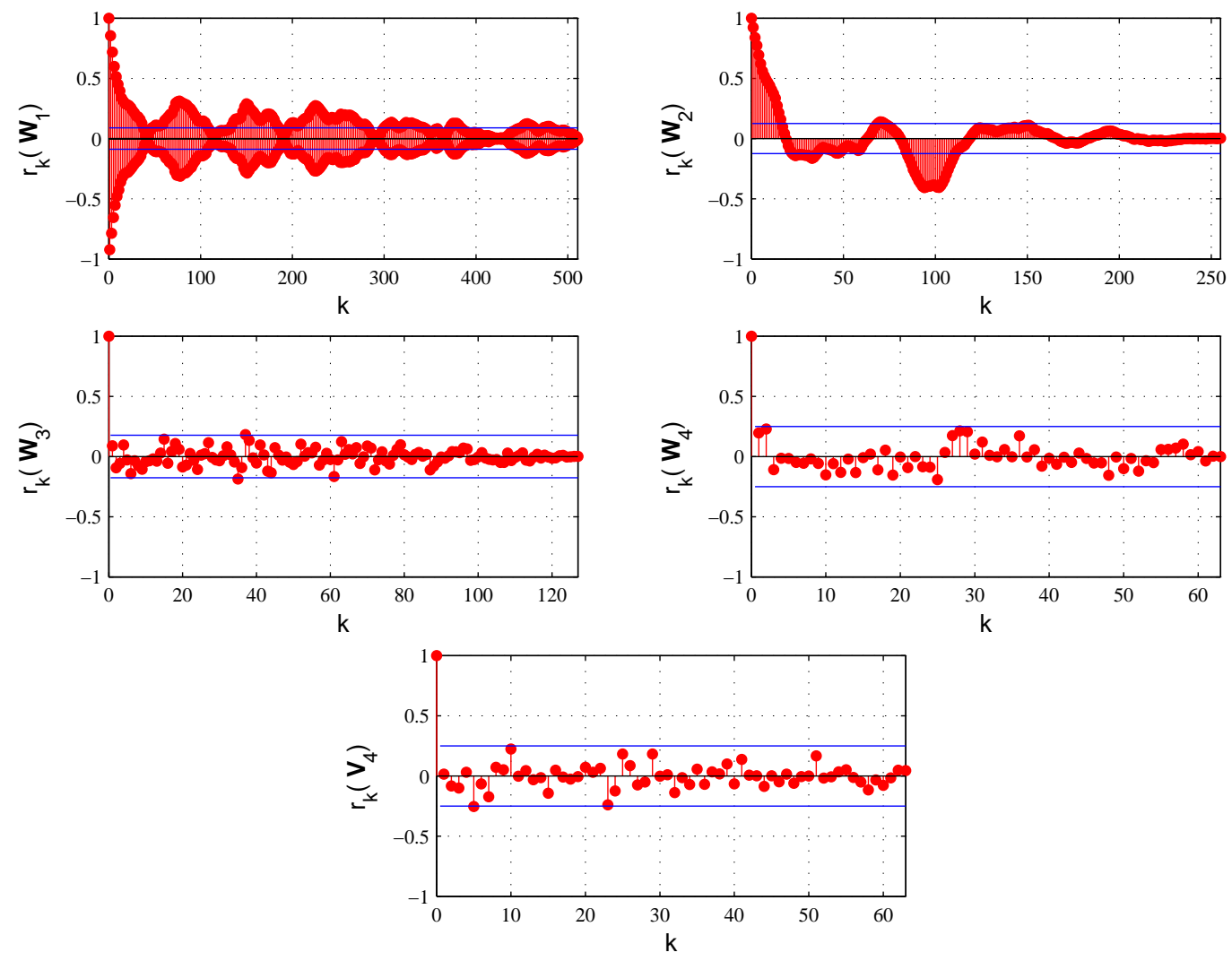

Figura 4.12: Autocorrelações dos coeficientes da decomposição DWT de $x_{2}(n)$ em 4 níveis utilizando filtros Daubechies de ordem 16.
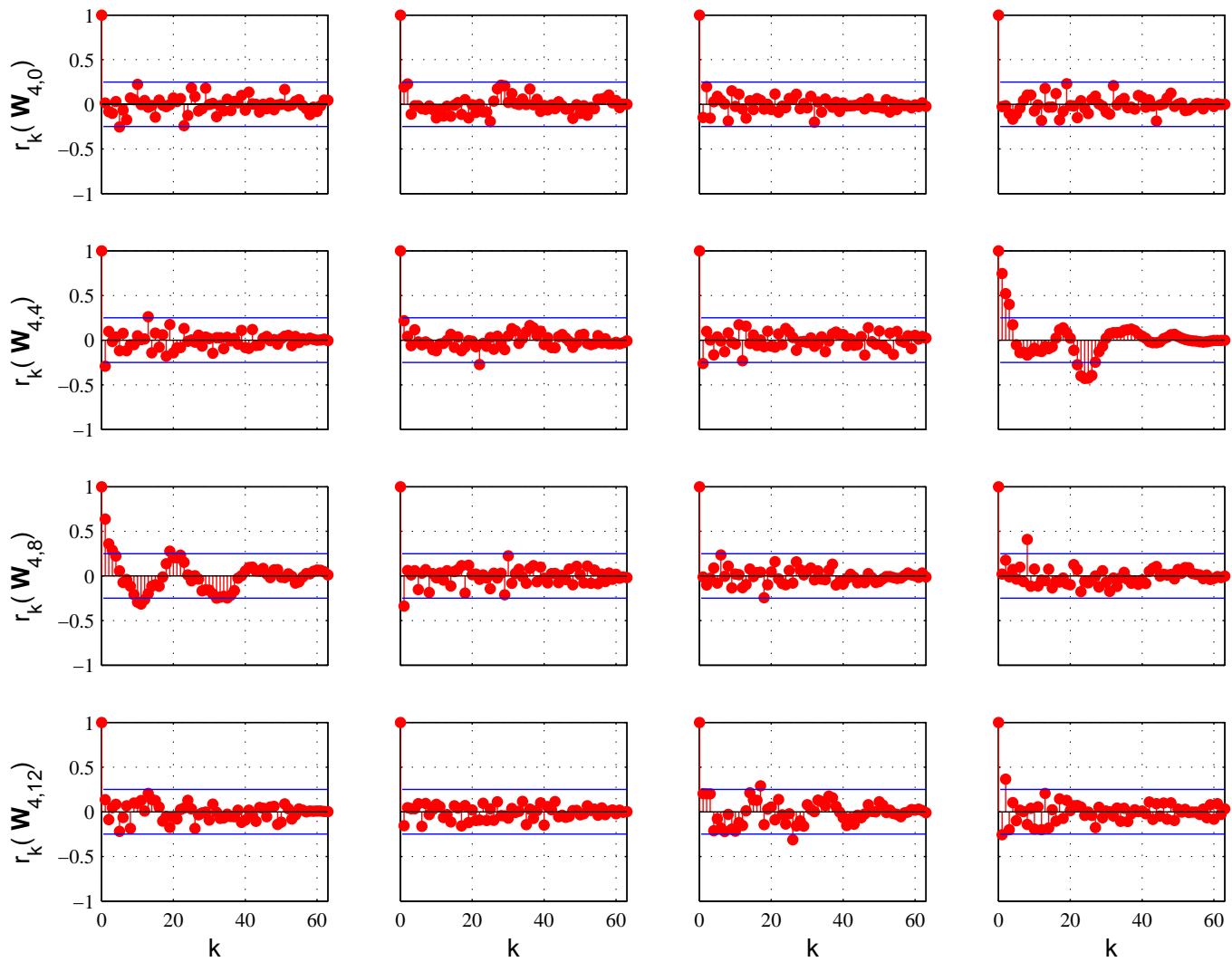

Figura 4.13: Autocorrelações dos coeficientes da decomposição DWPT de $x_{2}(n)$ em 4 níveis utilizando filtros Daubechies de ordem 16. 


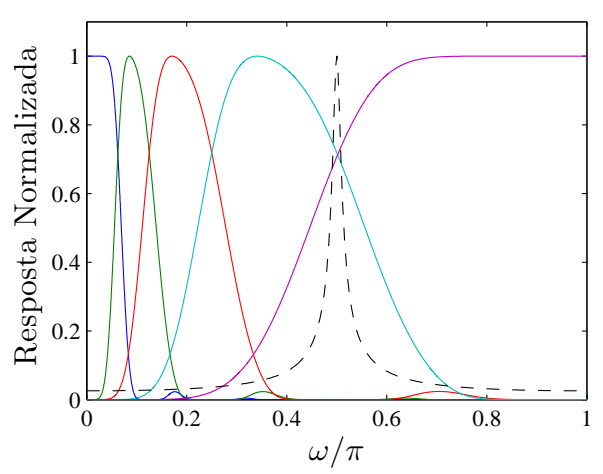

(a)

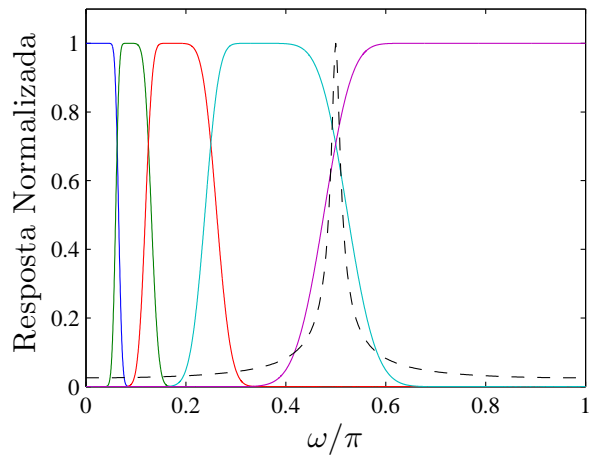

(b)

Figura 4.14: Resposta $S_{2 N}\left(e^{j \omega}\right)$ sobreposta à resposta dos filtros Daubechies, na decomposição DWT de 4 níveis, de ordem (a) 16; (b) 80.

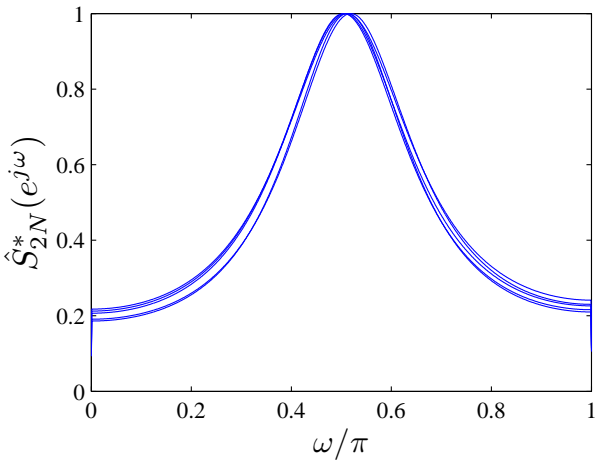

(a)

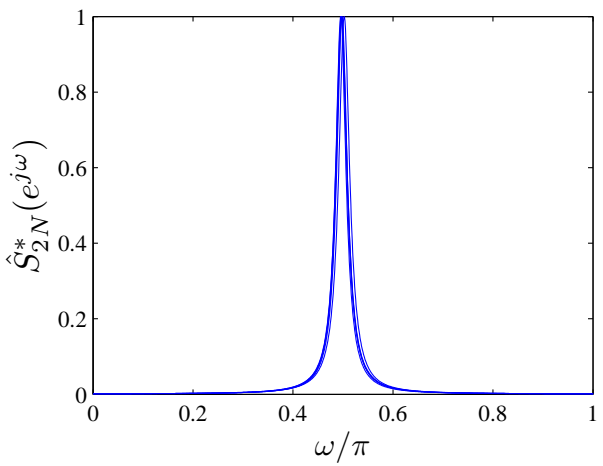

(b)

Figura 4.15: Estimativas de $S_{2 N}\left(e^{j \omega}\right)$ utilizando decomposições em 4 níveis com filtros Daubechies de ordem 16 dadas pelos procedimentos de (a) wavestrap; (b) wavestrapping.

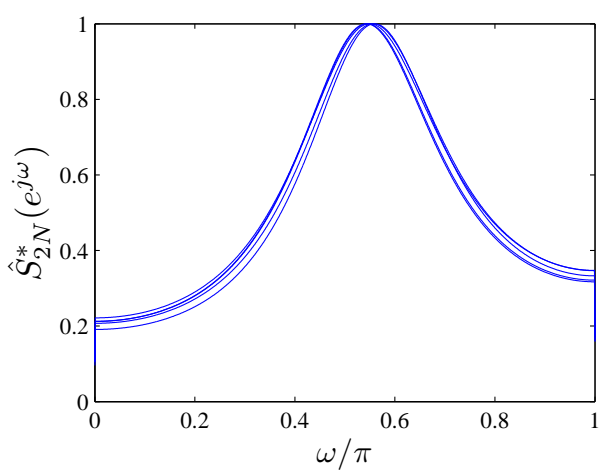

(a)

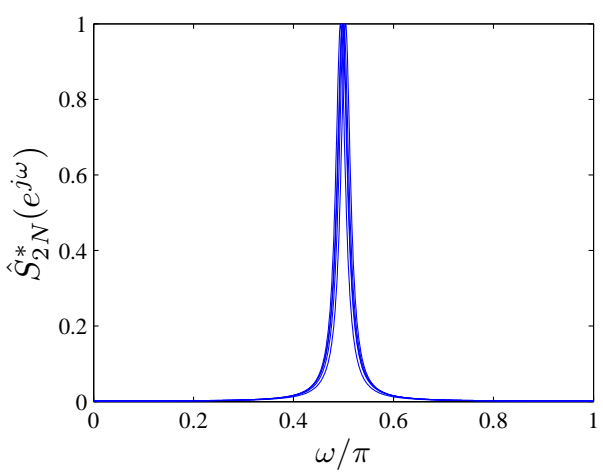

(b)

Figura 4.16: Estimativas de $S_{2 N}\left(e^{j \omega}\right)$ utilizando decomposições em 4 níveis com filtros Daubechies de ordem 80 dadas pelos procedimentos de (a) wavestrap; (b) wavestrapping. 
respostas do filtros Daubechies de ordem 16 sobrepostos à resposta $S_{2 N}\left(e^{j \omega}\right)$, onde pode-se ver que há um certo vazamento entre bandas, causando o resultado insatisfatório.

Se for aumentada a ordem dos filtros wavelet, por exemplo, de 16 para 80, e o processo for repetido (figura 4.16), é possível ver que ambos os resultados não melhoram significamente. Isto deve-se ao pico presente no espectro estar localizado exatamente numa região de transição dos filtros wavelet, conforme mostra a figura 4.14(b), tal que o aumento de ordem ainda não separa a potência do pico entre as bandas, fazendo com que o resultado não melhore.

Como último exemplo, é considerada a série $x_{3}(n)$ (figura 4.17), cujas autocorrelações dos coeficientes da decomposição em 4 níveis pelas DWT e DWPT com filtros Daubechies de ordem 20 são mostradas nas figuras 4.18 e 4.19, respectivamente. Desta vez, pode-se ver que os coeficientes presentes nos subvetores $\mathbf{W}_{1}$, $\mathbf{W}_{2}$ e $\mathbf{W}_{3}$ da DWT são correlacionados, assim como alguns coeficientes presentes nos subvetores da DWPT que aqui são considerados descorrelacionados.

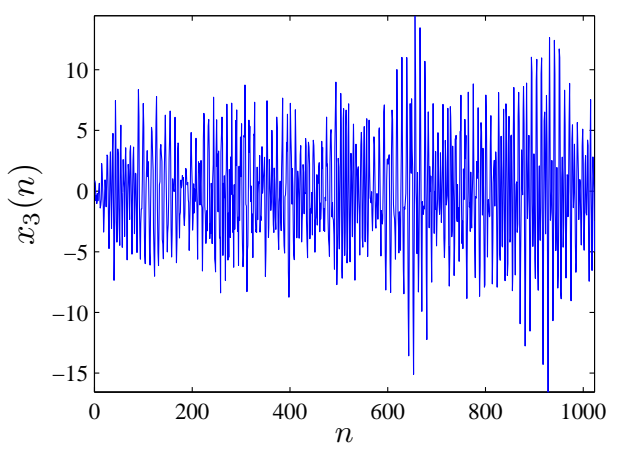

(a)

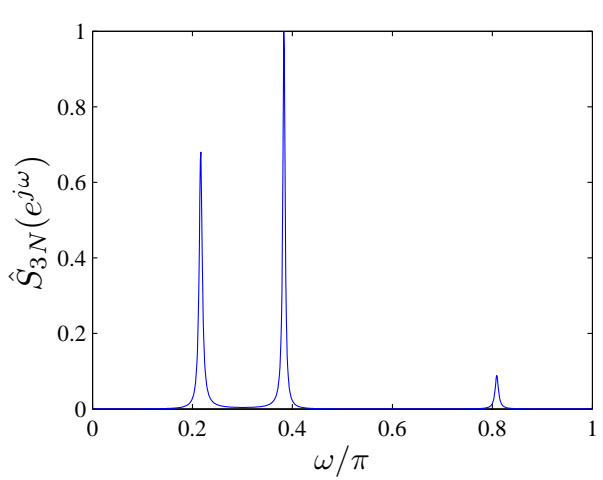

(b)

Figura 4.17: (a) Terceira série temporal $\left(x_{3}(n)\right)$; (b) Seu espectro de potência normalizado $\left(\hat{S}_{3 N}\left(e^{j \omega}\right)\right)$.

Aplicando 5 vezes os procedimentos estudados e determinando as estimativas espectrais (figura 4.21), vê-se que os resultados apresentados tanto pelo wavestrap quanto pelo wavestrapping são incoerentes com o esperado, que devem-se à forte correlação existente em grande parte dos subvetores. Além disto, se a ordem for aumentada, por exemplo, de 20 para 80, pouca melhoria será obtida na aplicação do wavestrap (figura 4.22(a)), visto que os picos ainda ficam separados cada um em uma banda (figura 4.20(b)), ao invés de serem repartidos entre elas.

Já para o wavestrapping, o aumento de ordem trouxe um resultado melhor (figura 4.22(b)), devido exatamente à potência dos picos ser particionada entre bandas adjacentes.

Sendo assim, com base nos três exemplos apresentados nesta seção, no que diz 

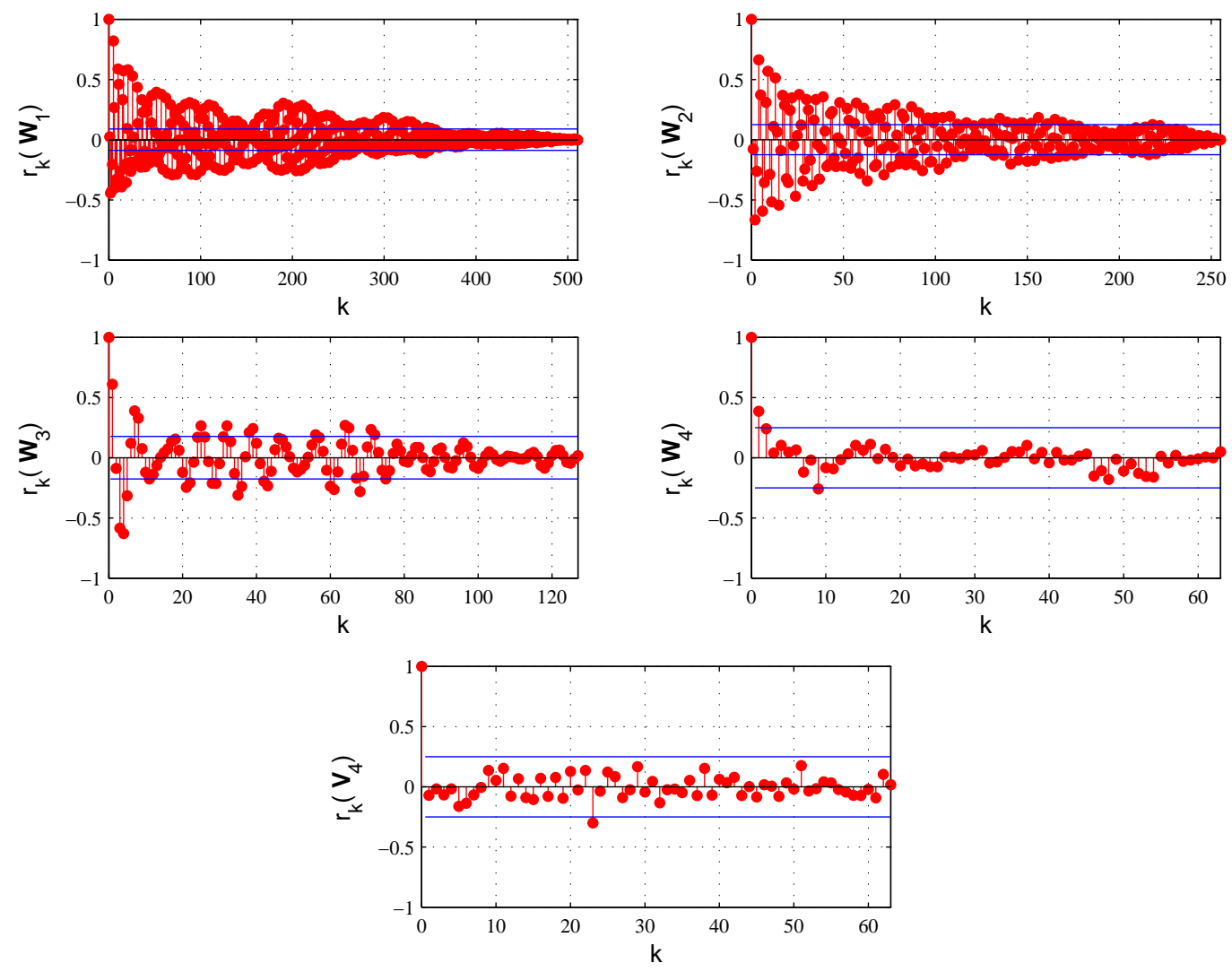

Figura 4.18: Autocorrelações dos coeficientes da decomposição DWT de $x_{3}(n)$ em 4 níveis utilizando filtros Daubechies de ordem 20.
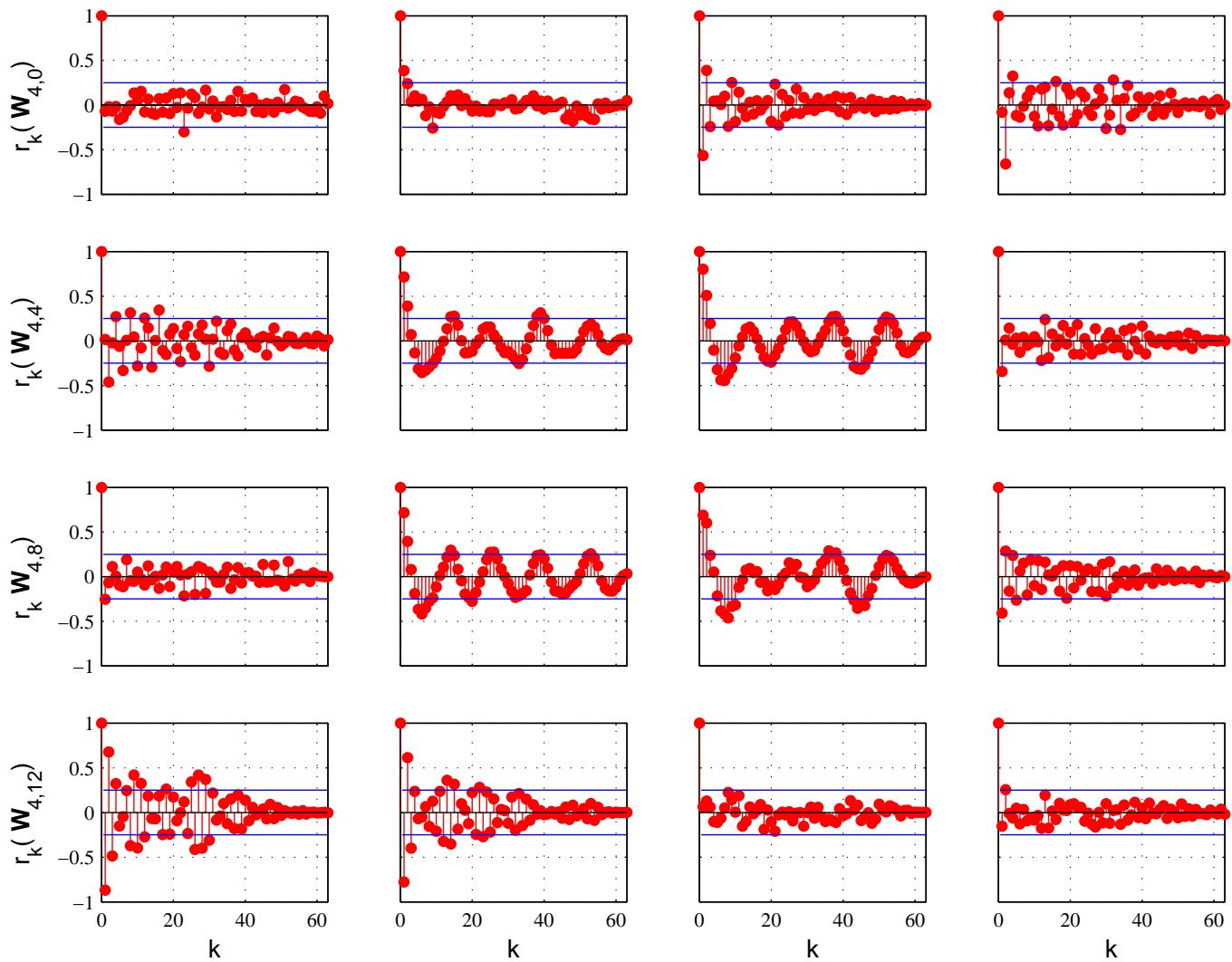

Figura 4.19: Autocorrelações dos coeficientes da decomposição DWPT de $x_{3}(n)$ em 4 níveis utilizando filtros Daubechies de ordem 20. 


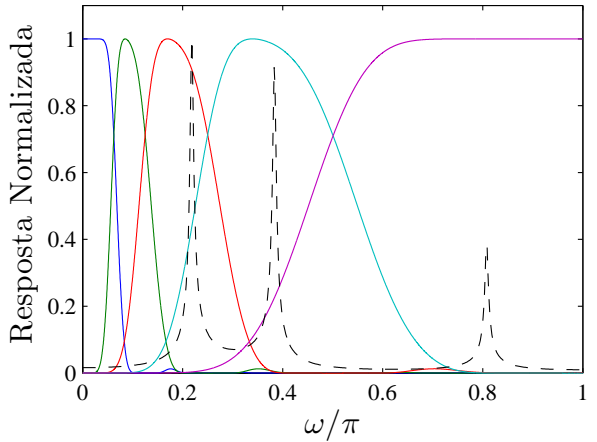

(a)

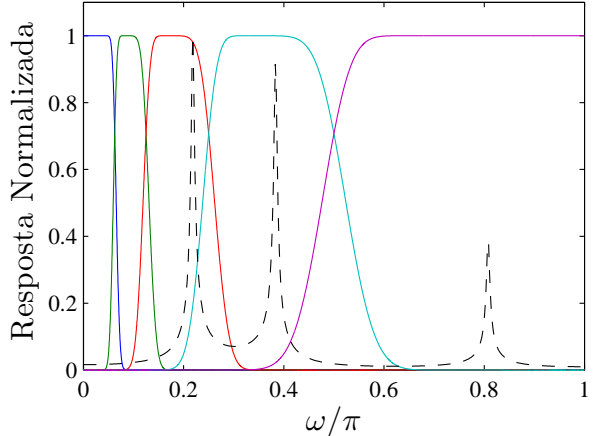

(b)

Figura 4.20: Resposta $S_{3 N}\left(e^{j \omega}\right)$ sobreposta à resposta dos filtros Daubechies, na decomposição DWT de 4 níveis, de ordem (a) 20; (b) 80.

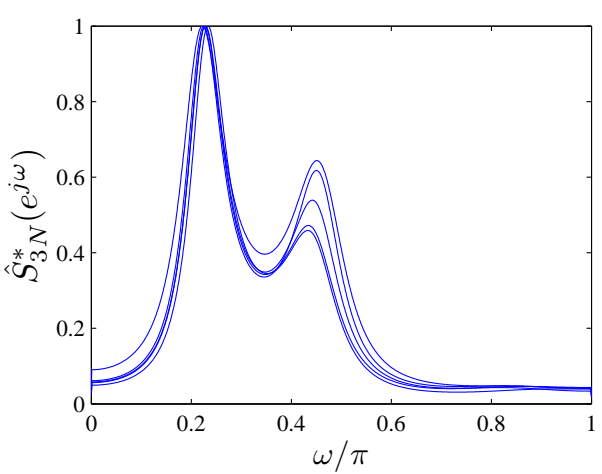

(a)

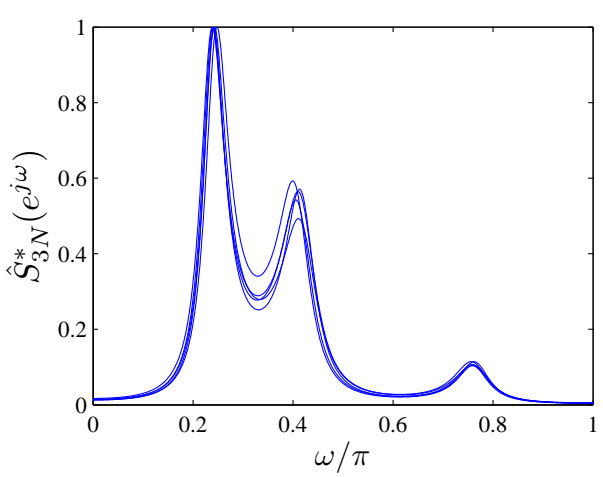

(b)

Figura 4.21: Estimativas de $S_{3 N}\left(e^{j \omega}\right)$ utilizando decomposições em 4 níveis com filtros Daubechies de ordem 20 dadas pelos procedimentos de (a) wavestrap; (b) wavestrapping.

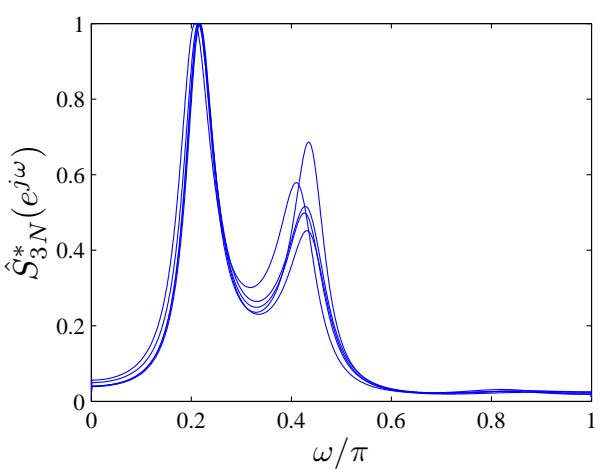

(a)

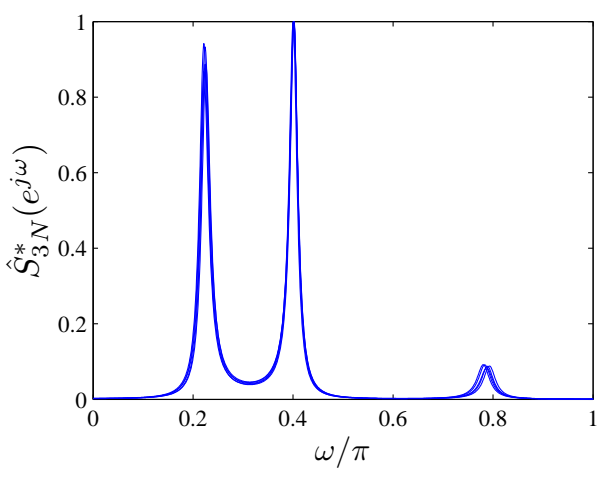

(b)

Figura 4.22: Estimativas de $S_{3 N}\left(e^{j \omega}\right)$ utilizando decomposições em 4 níveis com filtros Daubechies de ordem 80 dadas pelos procedimentos de (a) wavestrap; (b) 
respeito à ordem dos filtros utilizados na decomposição, é possível afirmar que o método baseado na DWPT é superior ao apresentado pela DWT, justamente pelo maior número de decomposições que a mesma é capaz de realizar no espectro.

\subsubsection{Considerações sobre o Número de Níveis na Decom- posição}

Nesta seção os procedimentos de wavestrap e wavestrapping são aplicados com $J_{0}=5$ e $L=80$. Tal alteração é feita na tentativa de se obterem resultados melhores quando estes métodos são aplicados sobre as séries $x_{2}(n)$ e $x_{3}(n)$.

Para a série $x_{2}(n)$ o problema é o pico acentuado presente em sua densidade espectral de potência $S_{2 N}\left(e^{j \omega}\right)$ na frequência $\omega=\pi / 2$, que é onde ocorre a transição dos filtros de decomposição wavelet.

Considerando novamente a série $x_{2}(n)$ (figura 4.11) porém, decompondo agora em 5 níveis com filtros Daubechies de ordem 80 (autocorrelações dos coeficientes DWT e DWPT mostradas nas figuras 4.23 e 4.24) tem-se, aplicando 5 vezes os procedimentos 5 e 6 , o resultado apresentado na figura 4.26 .

Comparando este resultado com o anterior (figura 4.16) é possível verificar que não houveram mudanças significativas tanto para o wavestrap como para o wavestrapping. Para o caso do wavestrap, isto pode ser explicado confrontando as figuras 4.14(b) e 4.25(a), onde pode-se ver que o aumento do número de decomposições não faz com que o cenário mude, ou seja, não faz com que a potência do pico seja distribuída. Além disto, pela natureza da DWT e por ter sido mantido $N=1024$, o fato de aumentar $J_{0}$ em uma unidade não faz com que os subvetores $\mathbf{W}_{1}$ e $\mathbf{W}_{2}$ sejam particionados sendo somente particionado o subvetor $\mathbf{V}_{4}$ (em $\mathbf{W}_{5}$ e $\mathbf{V}_{5}$ ), o que não resolve muito devido aos coeficientes presentes em $\mathbf{V}_{4}$ já serem descorrelacionados.

Já o novo resultado obtido com o wavestrapping (figura 4.26(b)) apresentou uma variabilidade menor que o anterior (figura 4.16(b)), devido ao espectro ser bem particionado por tal transformada (figura 4.25(b)).

Agora, considerando novamente a série $x_{3}(n)$ (figura 4.17) e decompondo em 5 níveis com filtros Daubechies de ordem 80 (autocorrelações dos coeficientes mostradas nas figuras 4.27 e 4.28), tem-se como resultado, repetindo 5 vezes os procedimentos 5 e 6 , o apresentado na figura 4.30 .

Comparando este resultado com o anterior (figura 4.22) novamente percebe-se uma pequena melhoria (menor variabilidade) para o wavestrapping, ao passo que 

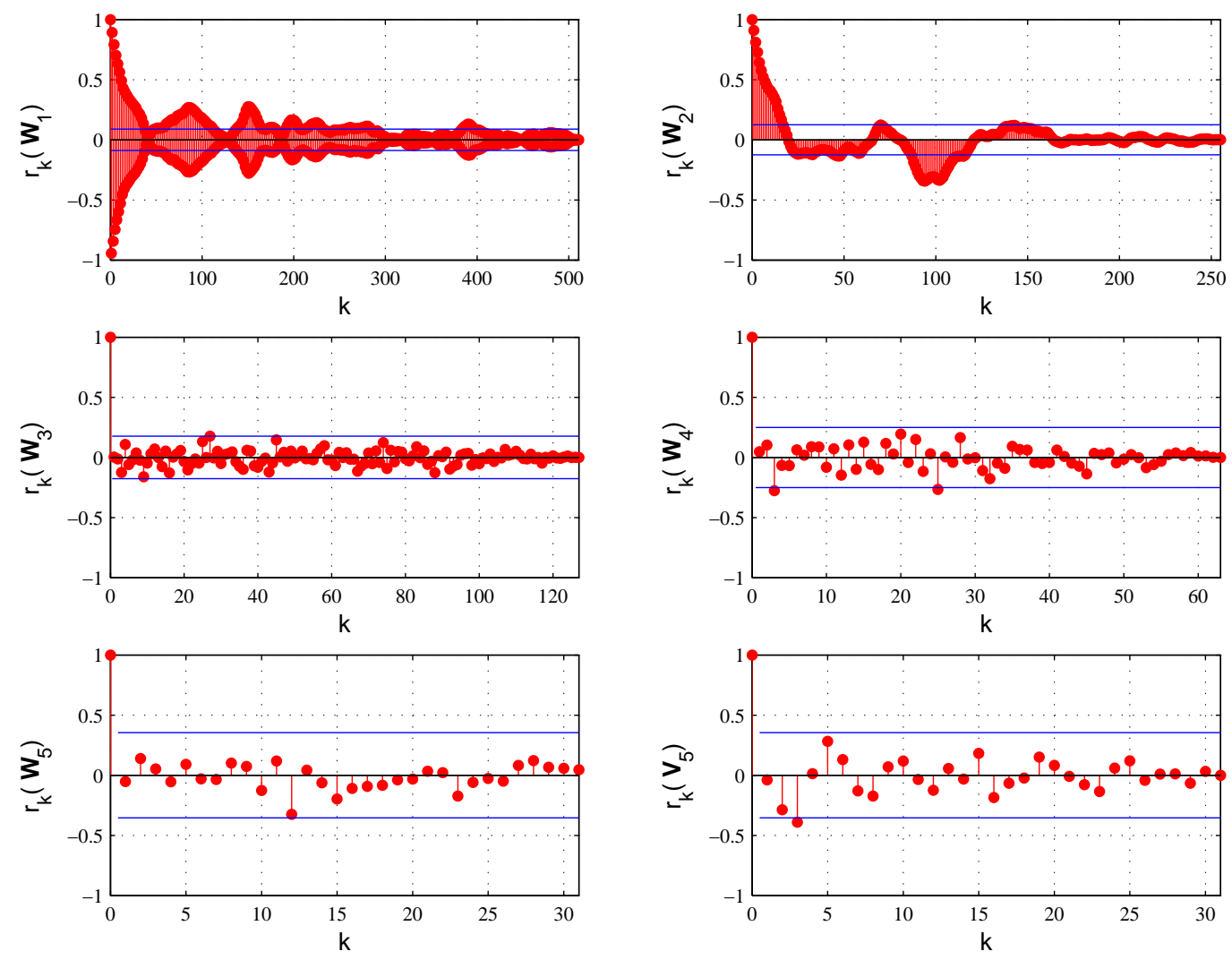

Figura 4.23: Autocorrelações dos coeficientes da decomposição DWT de $x_{2}(n)$ em 5 níveis utilizando filtros Daubechies de ordem 80.
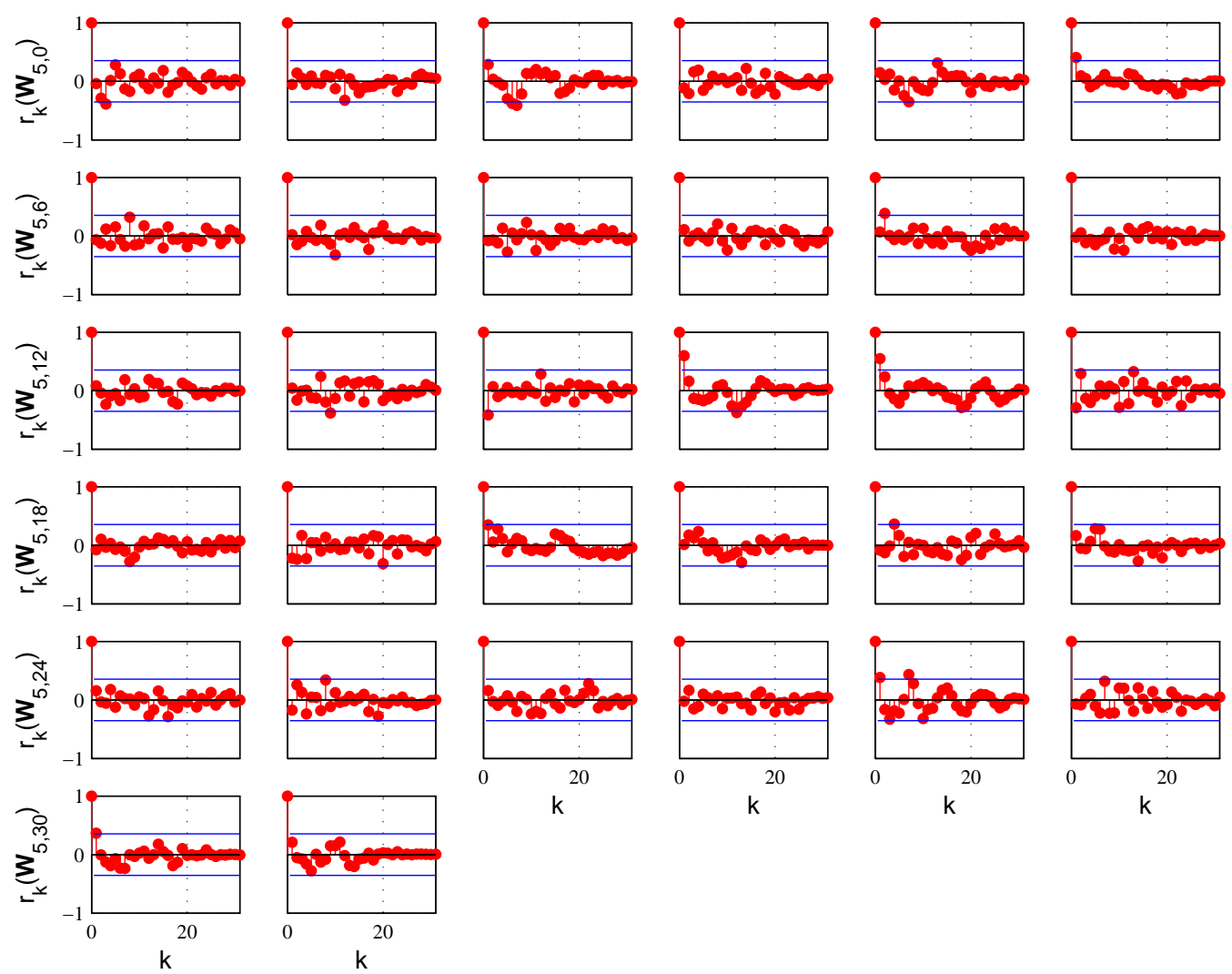

Figura 4.24: Autocorrelações dos coeficientes da decomposição DWPT de $x_{2}(n)$ em 5 níveis utilizando filtros Daubechies de ordem 80. 


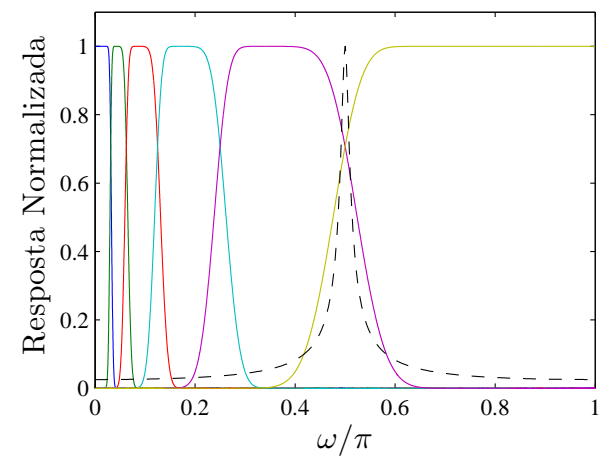

(a)

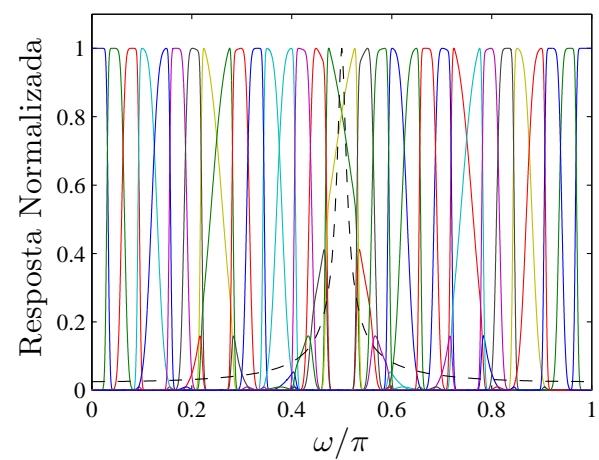

(b)

Figura 4.25: Resposta $S_{2 N}\left(e^{j \omega}\right)$ sobreposta à resposta dos filtros Daubechies de ordem 80, na decomposição em 5 níveis utilizando a (a) DWT; (b) DWPT.

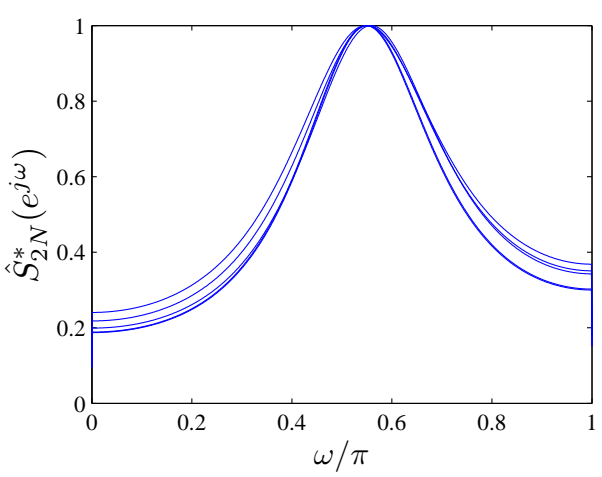

(a)

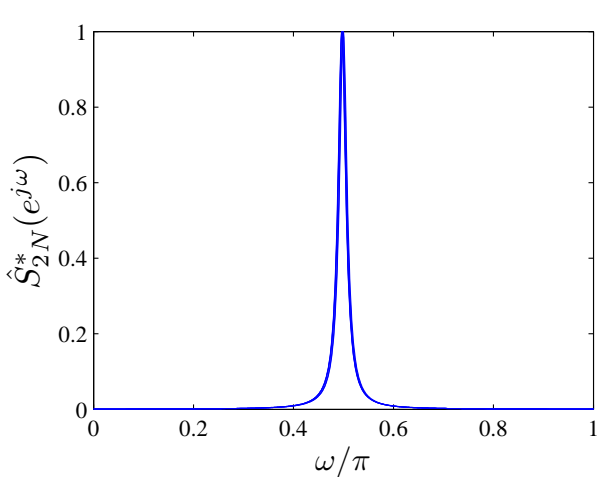

(b)

Figura 4.26: Estimativas de $S_{2 N}\left(e^{j \omega}\right)$ utilizando decomposições em 5 níveis com filtros Daubechies de ordem 80 dadas pelos procedimentos de (a) wavestrap; (b) wavestrapping.

para o wavestrap nada melhorou. Isto pode ser explicado confrontando as figuras 4.20(b) 4.29(a), onde pode-se ver que o fato de aumentar $J_{0}$ não faz com que a potência do espectro seja distribuída entre as bandas, e ainda, pela natureza da DWT e por ter sido mantido $N=1024$, o fato de aumentar o número de decomposições de 4 para 5 não faz com que os subvetores $\mathbf{W}_{1}, \mathbf{W}_{2}$ e $\mathbf{W}_{3}$ sejam particionados sendo somente particionado o subvetor $\mathbf{V}_{4}$, o que não resolve devido aos coeficientes presentes em $\mathbf{V}_{4}$ já estarem descorrelacionados.

Sendo assim, com base nestes exemplos apresentados e no que diz respeito ao número de níveis de decomposição, é possível afirmar que novamente o método baseado na DWPT apresenta resultados melhores comparados aos apresentados pela DWT, porém não muito melhores que os apresentados na seção anterior, onde foram consideradas alterações na ordem dos filtros de decomposição.

Além disto, é interessante notar que um cuidado deve ser tomado ao estudar a reamostragem wavelet quanto aos níveis de decomposição, ou seja, dado que $N=2^{J}$, se for escolhido $J_{0}$ muito próximo de $J$, sobrarão poucos coeficientes para serem reamostrados e não terá sentido aplicar os métodos de reamostragem 

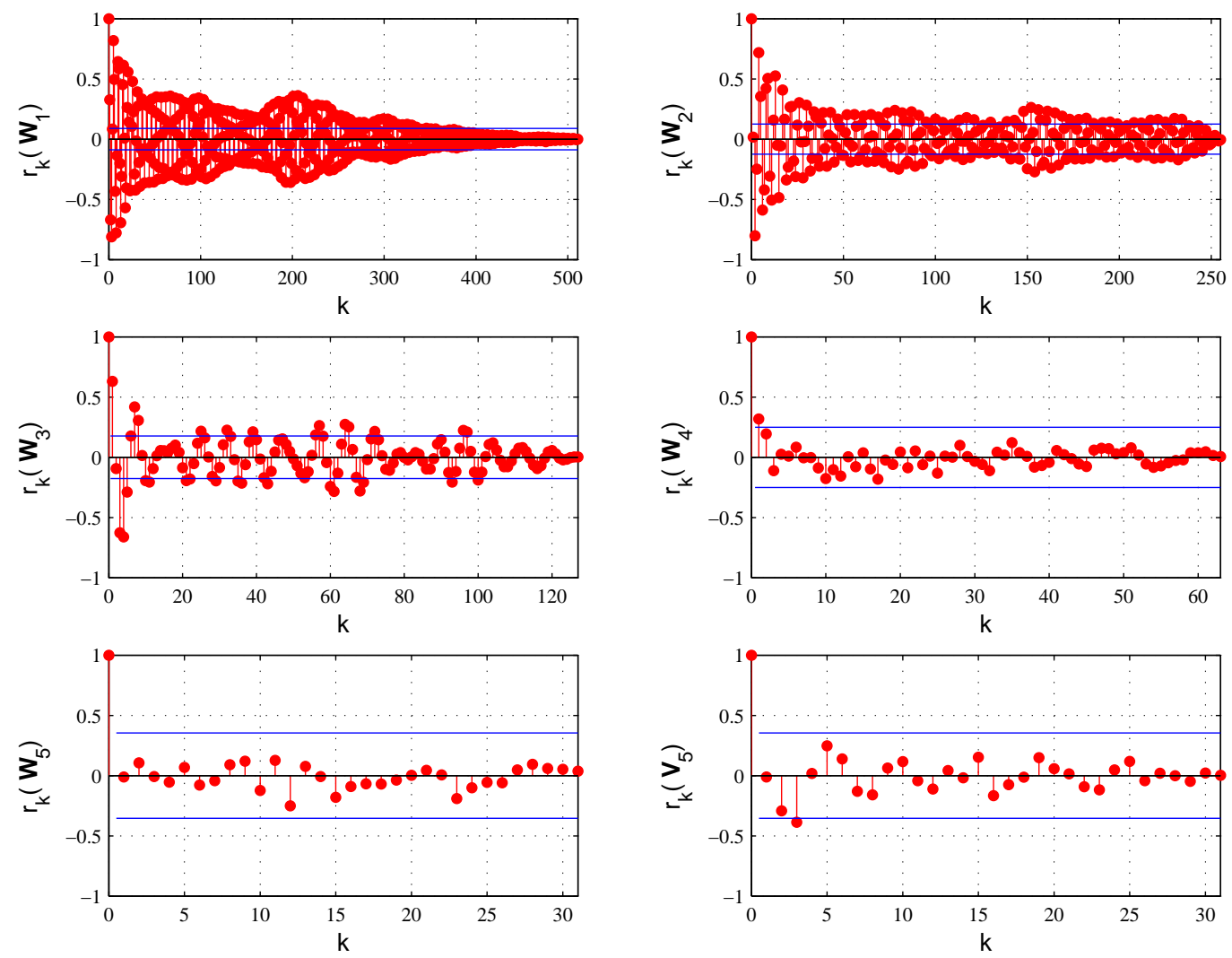

Figura 4.27: Autocorrelações dos coeficientes da decomposição DWT de $x_{3}(n)$ em 5 níveis utilizando filtros Daubechies de ordem 80.
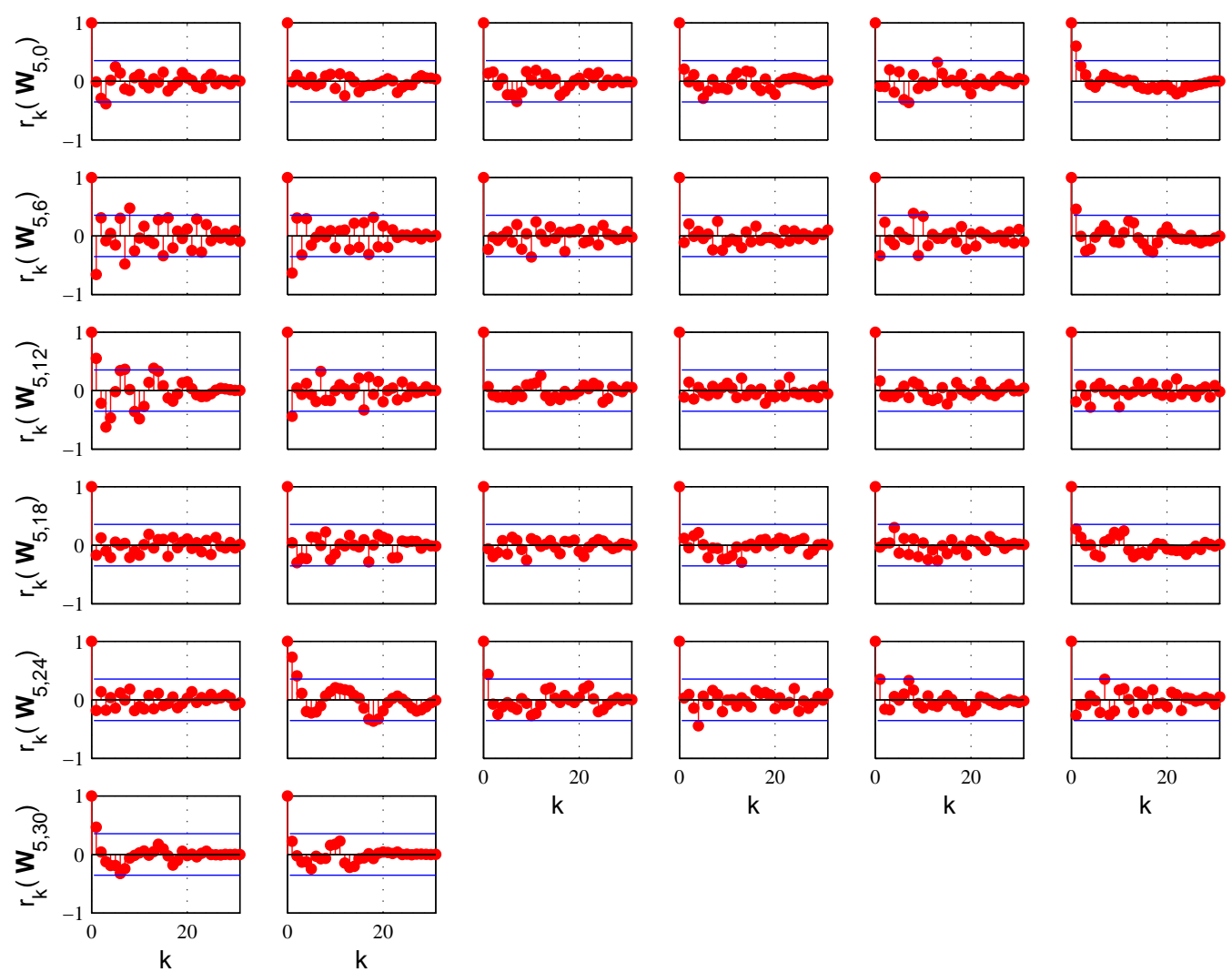

Figura 4.28: Autocorrelações dos coeficientes da decomposição DWPT de $x_{3}(n)$ em 5 níveis utilizando filtros Daubechies de ordem 80. 


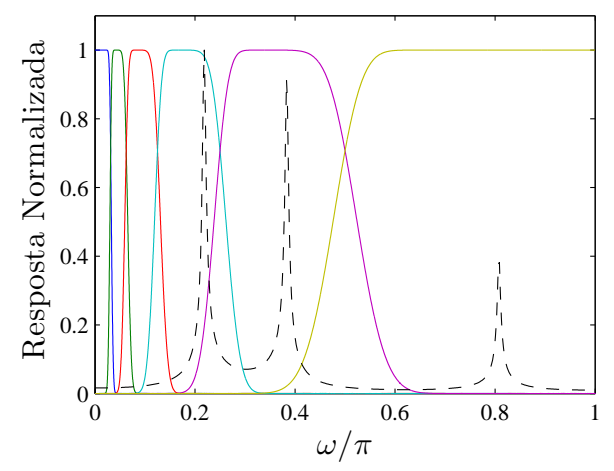

(a)

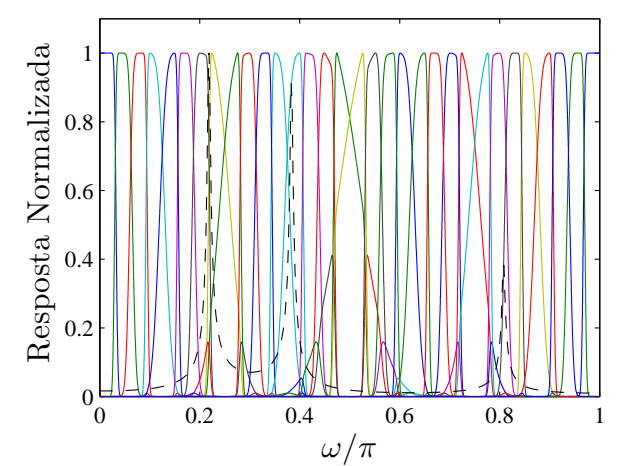

(b)

Figura 4.29: Resposta $S_{3 N}\left(e^{j \omega}\right)$ sobreposta à resposta dos filtros Daubechies de ordem 80, na decomposição em 5 níveis utilizando a (a) DWT; (b) DWPT.

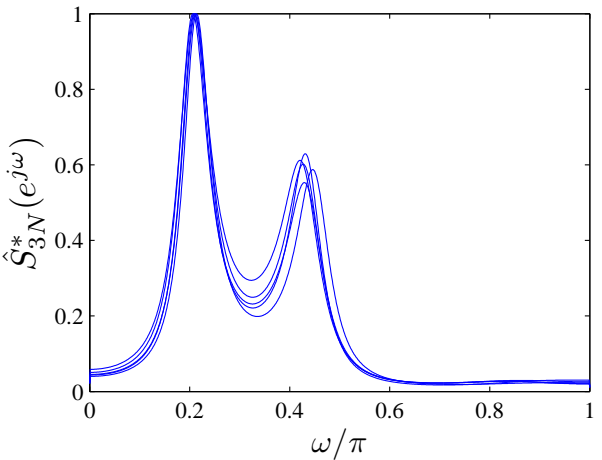

(a)

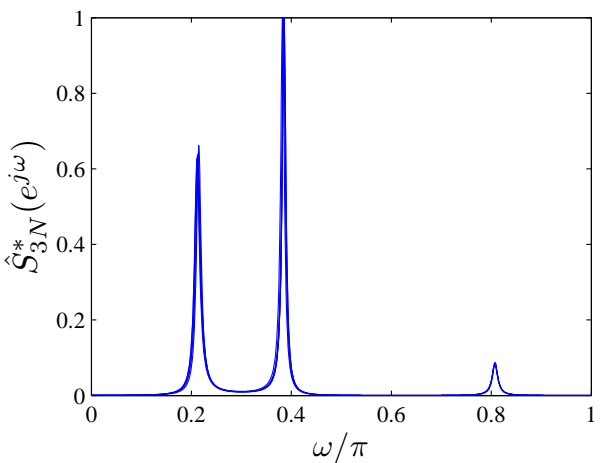

(b)

Figura 4.30: Estimativas de $S_{3 N}\left(e^{j \omega}\right)$ utilizando decomposições em 5 níveis com filtros Daubechies de ordem 80 dadas pelos procedimentos de (a) wavestrap; (b) wavestrapping.

baseados no domínio wavelet, sendo conveniente, nestes casos, àqueles que fazem o uso de blocos, mencionados no Capítulo 1. 


\section{Parte III}

\section{Conclusões}




\section{Conclusões e Trabalhos Futuros}

O presente trabalho investigou a utilidade da transformação wavelet de tempo discreto na reamostragem de séries temporais sintéticas geradas a partir de modelos AR com densidade espectral de potência conhecida. Para tanto, dois procedimentos foram apresentados e testados, o wavestrap e o wavestrapping baseados, respectivamente, nas DWT e DWPT, em três cenários diferentes quando: a ordem $L$ do filtro wavelet adotado é alterada; o número de níveis de decomposição $J_{0}$ é alterado; picos acentuados (polos próximos à circunferência unitária no plano z) estão presentes na representação espectral das séries temporais consideradas.

Nos três cenários propostos o wavestrapping foi superior ao wavestrap, devido ao fato de a DWPT decompor a banda $[\pi / 2, \pi]$, deixada de lado pela DWT.

Foi visto que, na presença de picos acentuados no espectro, para se ter uma reamostragem satisfatória por meio de wavelets, é necessário que a potência dos picos seja distribuída entre bandas adjacentes na decomposição, ou seja, não se pode ter picos isolados dentro de uma mesma faixa de frequências. Esta foi a maior observação feita neste estudo.

Desta forma, para se ter sucesso na reamostragem por wavelets, é preciso fazer uma avaliação do espectro do sinal que se deseja reamostrar e estabelecer um compromisso $L \times J_{0}$, de forma que a potência dos picos seja repartida entre bandas adjacentes com o menor vazamento possível.

Com relação ao número de níveis de decomposição $J_{0}$, é preciso tomar certo cuidado em determiná-lo devido ao comprimento do sinal $N=2^{J}$, isto é, se for escolhido $J_{0}$ muito próximo de $J$, não será coerente aplicar os métodos de reamostragem devido a se ter poucos coeficientes dentro de cada subvetor para se reamostrar. 


\subsection{Trabalhos Futuros}

A aplicação das wavelets como parte nos algoritmos de reamostragem ainda não está completamente esclarecida devido a várias questões que ainda estão sendo levantadas através de estudos exploratórios. Resta ainda, consolidar teoricamente os resultados obtidos por meio de demonstrações matemáticas que generalizem a utilidade das wavelets nos procedimentos de reamostragem.

Outro trabalho futuro está relacionado ao caminho utilizado para reconstruir a DWPT, ou seja, algum critério precisa ser estabelecido para se escolher os subvetores que serão utilizados na reconstrução.

Um estudo comparativo entre os métodos de wavestrapping e Monte Carlo também é um tópico interessante de ser trabalhado.

Finalmente, um último trabalho seria agrupar os principais resultados e, só então, aplicar o estudo sobre séries temporais reais, tais como sinais provenientes de exames de EEG e fMRI. 


\section{Anexo A - Banco de Filtros: Considerações sobre Reconstrução}

Este anexo tem por objetivo mostrar que o banco de fltros utilizado pela DWT e DWPT, mostrado na figura A.1 permite a reconstrução perfeita sem atraso, ou seja, é mostrado que $\tilde{X}(z)=X(z)$.

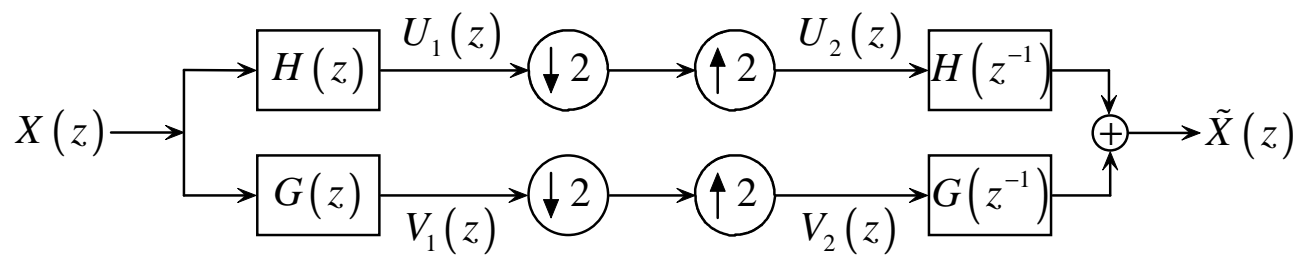

Figura A.1: Banco de filtros para decomposição wavelet.

Temos que,

$$
U_{1}(z)=H(z) X(z) \quad \text { e } \quad V_{1}(z)=G(z) X(z)
$$

De acordo com [23],

$$
\begin{aligned}
& U_{2}(z)=\frac{1}{2}\left[U_{1}(z)+U_{1}(-z)\right]=\frac{1}{2}[H(z) X(z)+H(-z) X(-z)] \\
& V_{2}(z)=\frac{1}{2}\left[V_{1}(z)+V_{1}(-z)\right]=\frac{1}{2}[G(z) X(z)+G(-z) X(-z)]
\end{aligned}
$$

Desta forma

$$
\tilde{X}(z)=U_{2}(z) H\left(z^{-1}\right)+V_{2}(z) G\left(z^{-1}\right)=\frac{1}{2} A(z) X(z)+\frac{1}{2} B(z) X(-z)
$$

tal que,

$$
A(z)=H(z) H\left(z^{-1}\right)+G(z) G\left(z^{-1}\right)
$$

e

$$
B(z)=H(-z) H\left(z^{-1}\right)+G(-z) G\left(z^{-1}\right)
$$

Assim, para se conseguir a reconstrução perfeita sem atraso, ou seja, para 
que $\tilde{X}(z)=X(z)$, é preciso que

$$
B(z)=0 \quad \text { e } \quad A(z)=2
$$

O que, de fato, é alcançado fazendo

$$
G(z)=z^{-(L-1)} H\left(-z^{-1}\right) .
$$

onde $L$ é um número par representando o comprimento dos filtros.

A verificação das condições A.6 é imediata por meio das seguintes derivações de A.7,

$$
G(-z)=-z^{-(L-1)} H\left(z^{-1}\right) \quad \text { e } \quad G\left(z^{-1}\right)=z^{L-1} H(-z)
$$

No domínio do tempo, a equação A.7 tem a forma,

$$
g(n)=(-1)^{n+1} h(L-1-n)
$$

e sendo assim, os filtros $H(z)$ e $G(z)$ são ditos estar em quadratura. 


\section{Anexo B - Decomposição Wavelet: Construção da Matriz $\mathcal{W}$}

Neste anexo serão apresentados exemplos de decomposição wavelet para as transformadas DWT e DWPT, ambas de tempo discreto. Para tanto, será considerado o seguinte sinal

$$
\left.\mathbf{x}=\begin{array}{cccccccc}
0,2 & -0,4 & -0,6 & -0,5 & -0,8 & -0,4 & -0,9 & 0,0 \\
-0,2 & 0,1 & -0,1 & 0,1 & 0,7 & 0,9 & 0,0 & 0,3
\end{array}\right]
$$

apresentado na figura B.1.

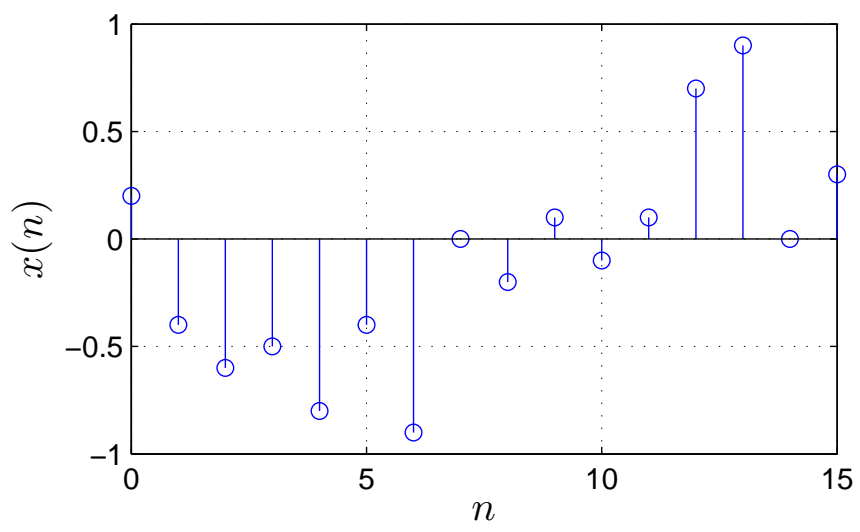

Figura B.1: Sinal $x(n)$ de comprimento $N=16$.

Para um sinal

$$
\mathbf{x}=[x(0) \cdots x(N-1)]^{T},
$$

com $N=2^{J}$ o algoritmo piramidal de Mallat fornece os coeficientes DWT

$$
\mathbf{W}=\mathcal{W} \mathbf{x}
$$


depois de $J_{0}$ repetições através da equação de análise,

$$
\mathbf{W}=\left[\begin{array}{c}
\mathbf{W}_{1} \\
\mathbf{W}_{2} \\
\vdots \\
\mathbf{W}_{j} \\
\vdots \\
\mathbf{W}_{J_{0}} \\
\mathbf{V}_{J_{0}}
\end{array}\right]=\left[\begin{array}{c}
\mathcal{W}_{1} \\
\mathcal{W}_{2} \\
\vdots \\
\mathcal{W}_{j} \\
\vdots \\
\mathcal{W}_{J_{0}} \\
\mathcal{W}_{J_{0}}
\end{array}\right] \mathbf{x}=\left[\begin{array}{c}
\mathcal{B}_{1} \\
\mathcal{B}_{2} \mathcal{A}_{1} \\
\vdots \\
\mathcal{B}_{j} \mathcal{A}_{j-1} \cdots \mathcal{A}_{1} \\
\vdots \\
\mathcal{B}_{J_{0}} \mathcal{A}_{J_{0}-1} \cdots \mathcal{A}_{1} \\
\mathcal{A}_{J_{0}} \mathcal{A}_{J_{0}-1} \cdots \mathcal{A}_{1}
\end{array}\right] \mathbf{x}
$$

onde $\mathcal{B}_{j}$ e $\mathcal{A}_{j}$ são matrizes de ordem $N_{j} \times N_{j-1}$, com $N_{j}=\frac{N}{2^{j}}$, onde as linhas contêm, respectivamente, deslocamentos circulares dos filtros wavelet e escala $\{h(n): n=0, \cdots, L-1\}$ e $\{g(n): n=0, \cdots, L-1\}$ periodizados para o comprimento $N_{j-1}$.

Com estas novas matrizes $\mathcal{B}_{j}$ e $\mathcal{A}_{j}$ é possível visualizar a decomposição por meio de uma pirâmide, conforme mostra a figura B.2 para o caso de $J_{0}=3$.

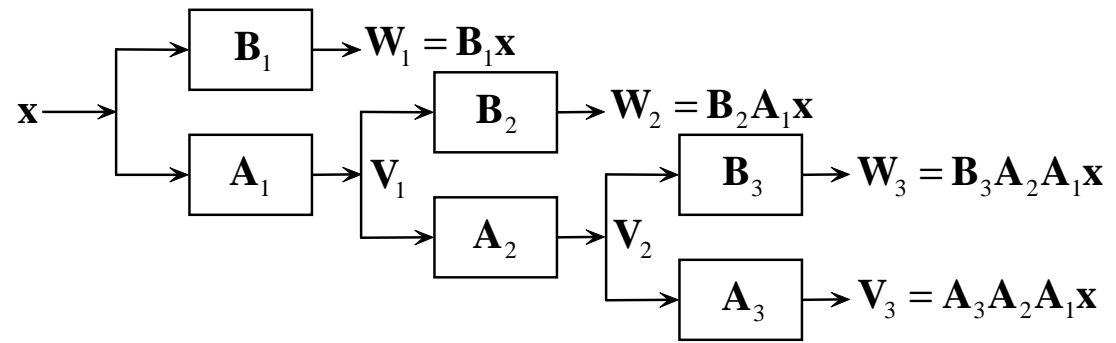

Figura B.2: Árvore matricial para a DWT com $J_{0}=3$.

Como exemplo, é decomposto o sinal $x(n)$ mostrado na figura B.1 em $J_{0}=3$ níveis,

$$
\mathbf{W}=\mathcal{W} \mathbf{x}=\left[\begin{array}{c}
\mathcal{B}_{1} \\
\mathcal{B}_{2} \mathcal{A}_{1} \\
\mathcal{B}_{3} \mathcal{A}_{2} \mathcal{A}_{1} \\
\mathcal{A}_{3} \mathcal{A}_{2} \mathcal{A}_{1}
\end{array}\right] \mathbf{x}
$$

utilizando filtros Daubechies de ordem 4 cujos coeficientes são

$$
h_{0}=\frac{1-\sqrt{3}}{4 \sqrt{2}}, \quad h_{1}=\frac{-3+\sqrt{3}}{4 \sqrt{2}}, \quad h_{2}=\frac{3+\sqrt{3}}{4 \sqrt{2}}, \quad h_{3}=\frac{-1-\sqrt{3}}{4 \sqrt{2}} .
$$

Para este caso as matrizes $\mathcal{B}_{j}$ e $\mathcal{A}_{j}$ são as seguintes 


$$
\mathcal{B}_{1}=\left[\begin{array}{cccccccccccccccc}
h_{1} & h_{0} & 0 & 0 & 0 & 0 & 0 & 0 & 0 & 0 & 0 & 0 & 0 & 0 & h_{3} & h_{2} \\
h_{3} & h_{2} & h_{1} & h_{0} & 0 & 0 & 0 & 0 & 0 & 0 & 0 & 0 & 0 & 0 & 0 & 0 \\
0 & 0 & h_{3} & h_{2} & h_{1} & h_{0} & 0 & 0 & 0 & 0 & 0 & 0 & 0 & 0 & 0 & 0 \\
0 & 0 & 0 & 0 & h_{3} & h_{2} & h_{1} & h_{0} & 0 & 0 & 0 & 0 & 0 & 0 & 0 & 0 \\
0 & 0 & 0 & 0 & 0 & 0 & h_{3} & h_{2} & h_{1} & h_{0} & 0 & 0 & 0 & 0 & 0 & 0 \\
0 & 0 & 0 & 0 & 0 & 0 & 0 & 0 & h_{3} & h_{2} & h_{1} & h_{0} & 0 & 0 & 0 & 0 \\
0 & 0 & 0 & 0 & 0 & 0 & 0 & 0 & 0 & 0 & h_{3} & h_{2} & h_{1} & h_{0} & 0 & 0 \\
0 & 0 & 0 & 0 & 0 & 0 & 0 & 0 & 0 & 0 & 0 & 0 & h_{3} & h_{2} & h_{1} & h_{0}
\end{array}\right]
$$$$
\mathcal{A}_{1}=\left[\begin{array}{cccccccccccccccc}
g_{1} & g_{0} & 0 & 0 & 0 & 0 & 0 & 0 & 0 & 0 & 0 & 0 & 0 & 0 & g_{3} & g_{2} \\
g_{3} & g_{2} & g_{1} & g_{0} & 0 & 0 & 0 & 0 & 0 & 0 & 0 & 0 & 0 & 0 & 0 & 0 \\
0 & 0 & g_{3} & g_{2} & g_{1} & g_{0} & 0 & 0 & 0 & 0 & 0 & 0 & 0 & 0 & 0 & 0 \\
0 & 0 & 0 & 0 & g_{3} & g_{2} & g_{1} & g_{0} & 0 & 0 & 0 & 0 & 0 & 0 & 0 & 0 \\
0 & 0 & 0 & 0 & 0 & 0 & g_{3} & g_{2} & g_{1} & g_{0} & 0 & 0 & 0 & 0 & 0 & 0 \\
0 & 0 & 0 & 0 & 0 & 0 & 0 & 0 & g_{3} & g_{2} & g_{1} & g_{0} & 0 & 0 & 0 & 0 \\
0 & 0 & 0 & 0 & 0 & 0 & 0 & 0 & 0 & 0 & g_{3} & g_{2} & g_{1} & g_{0} & 0 & 0 \\
0 & 0 & 0 & 0 & 0 & 0 & 0 & 0 & 0 & 0 & 0 & 0 & g_{3} & g_{2} & g_{1} & g_{0}
\end{array}\right]
$$

$$
\begin{gathered}
\mathcal{B}_{2}=\left[\begin{array}{cccccccc}
h_{1} & h_{0} & 0 & 0 & 0 & 0 & h_{3} & h_{2} \\
h_{3} & h_{2} & h_{1} & h_{0} & 0 & 0 & 0 & 0 \\
0 & 0 & h_{3} & h_{2} & h_{1} & h_{0} & 0 & 0 \\
0 & 0 & 0 & 0 & h_{3} & h_{2} & h_{1} & h_{0}
\end{array}\right] \\
\mathcal{A}_{2}=\left[\begin{array}{cccccccc}
g_{1} & g_{0} & 0 & 0 & 0 & 0 & g_{3} & g_{2} \\
g_{3} & g_{2} & g_{1} & g_{0} & 0 & 0 & 0 & 0 \\
0 & 0 & g_{3} & g_{2} & g_{1} & g_{0} & 0 & 0 \\
0 & 0 & 0 & 0 & g_{3} & g_{2} & g_{1} & g_{0}
\end{array}\right] \\
\mathcal{B}_{3}=\left[\begin{array}{lllllll}
h_{1} & h_{0} & h_{3} & h_{2} \\
h_{3} & h_{2} & h_{1} & h_{0}
\end{array}\right] \mathcal{A}_{3}=\left[\begin{array}{llll}
g_{1} & g_{0} & g_{3} & g_{2} \\
g_{3} & g_{2} & g_{1} & g_{0}
\end{array}\right]
\end{gathered}
$$

Os detalhes para a construção de tais matrizes podem ser encontrados em [11] que aqui são omitidos devido a grande teoria envolvida e ao foco do trabalho. Desta forma, os coeficientes são os seguintes 


$$
\begin{gathered}
\mathbf{W}_{1}=\mathcal{B}_{1} \mathbf{x}=\left[\begin{array}{r}
0,25789 \\
-0,23201 \\
0,10260 \\
0,25349 \\
0,46655 \\
0,18972 \\
-0,14142 \\
0,37597
\end{array}\right] \\
\mathbf{W}_{2}=\mathcal{B}_{2} \mathcal{A}_{1} \mathbf{x}=\left[\begin{array}{r}
-0,19375 \\
-0,44183 \\
-0,18615 \\
-0,25768
\end{array}\right] \\
\mathbf{W}_{3}=\mathcal{B}_{3} \mathcal{A}_{2} \mathcal{A}_{1} \mathbf{x}=\left[\begin{array}{r}
1,13861 \\
-0,99025
\end{array}\right], \\
\mathbf{V}_{3}=\mathcal{A}_{3} \mathcal{A}_{2} \mathcal{A}_{1} \mathbf{x}=\left[\begin{array}{r}
-0,78235 \\
0,21667
\end{array}\right]
\end{gathered}
$$

A reconstrução da série $x(n)$ utilizando as matrizes $\mathcal{B}_{j}$ e $\mathcal{A}_{j}$ pode ser visualizada na figura B.3.

$$
\mathbf{x}=\mathbf{B}_{1}^{T} \mathbf{W}_{1}+\mathbf{A}_{1}^{T} \mathbf{B}_{2}^{T} \mathbf{W}_{2}+\mathbf{A}_{1}^{T} \mathbf{A}_{2}^{T} \mathbf{B}_{3}^{T} \mathbf{W}_{3}+\mathbf{A}_{1}^{T} \mathbf{A}_{2}^{T} \mathbf{A}_{3}^{T} \mathbf{V}_{3}
$$

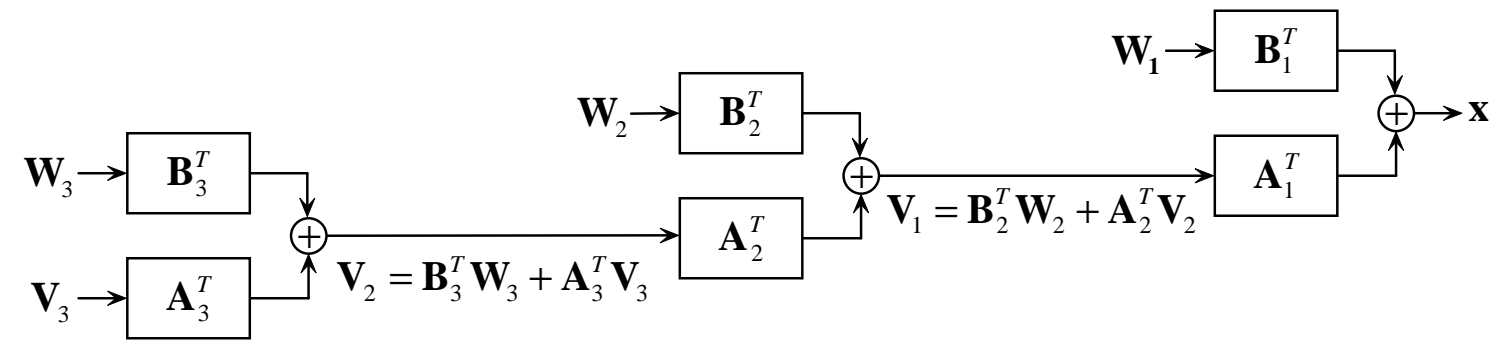

Figura B.3: Árvore matricial inversa para a DWT com $J_{0}=3$.

Para a decomposição utilizando a DWPT o processo é semelhante e as matrizes $\mathcal{B}_{j}$ e $\mathcal{A}_{j}$ são as mesmas, mudando somente a ordem das multiplicações, conforme ilustra a figura B.4. 


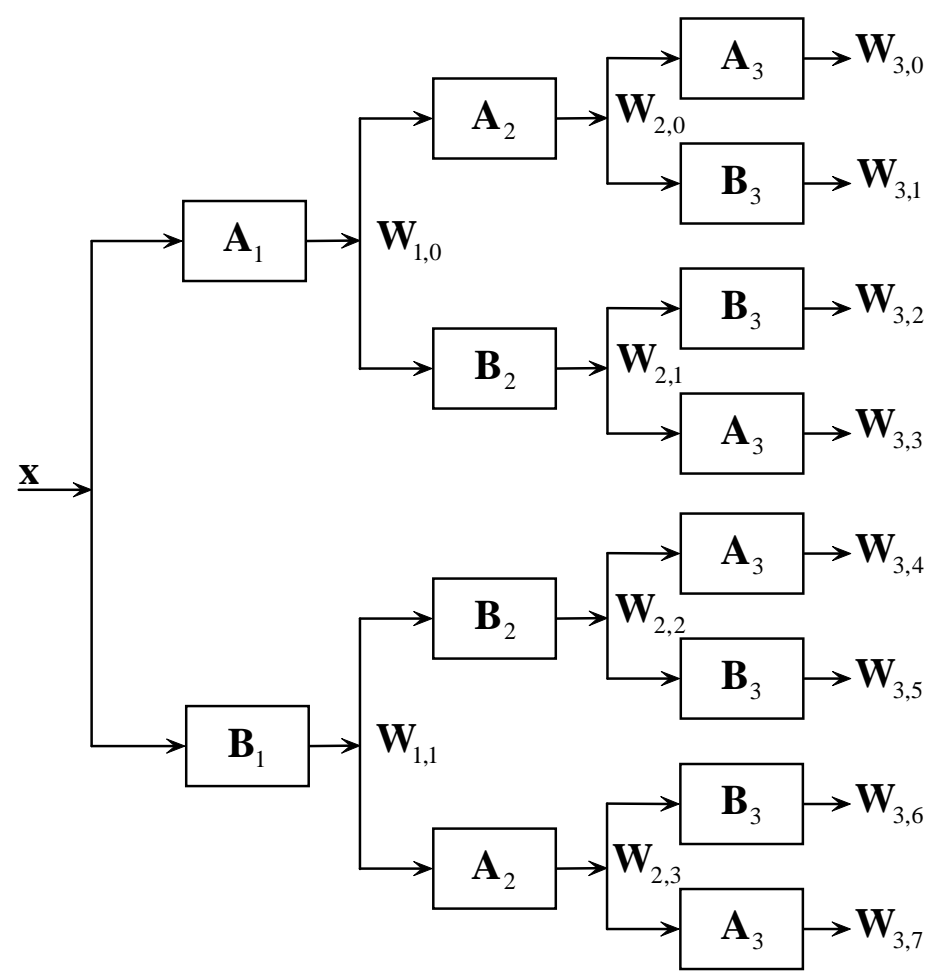

Figura B.4: Árvore matricial para a DWPT com $J_{0}=3$. 


\section{Anexo C - Códigos MATLAB}

Neste anexo, são disponibilizadas as rotinas utilizadas para decomposição DWT e DWPT de tempo discreto. Elas são aplicáveis sempre que os sinais têm duração finita.

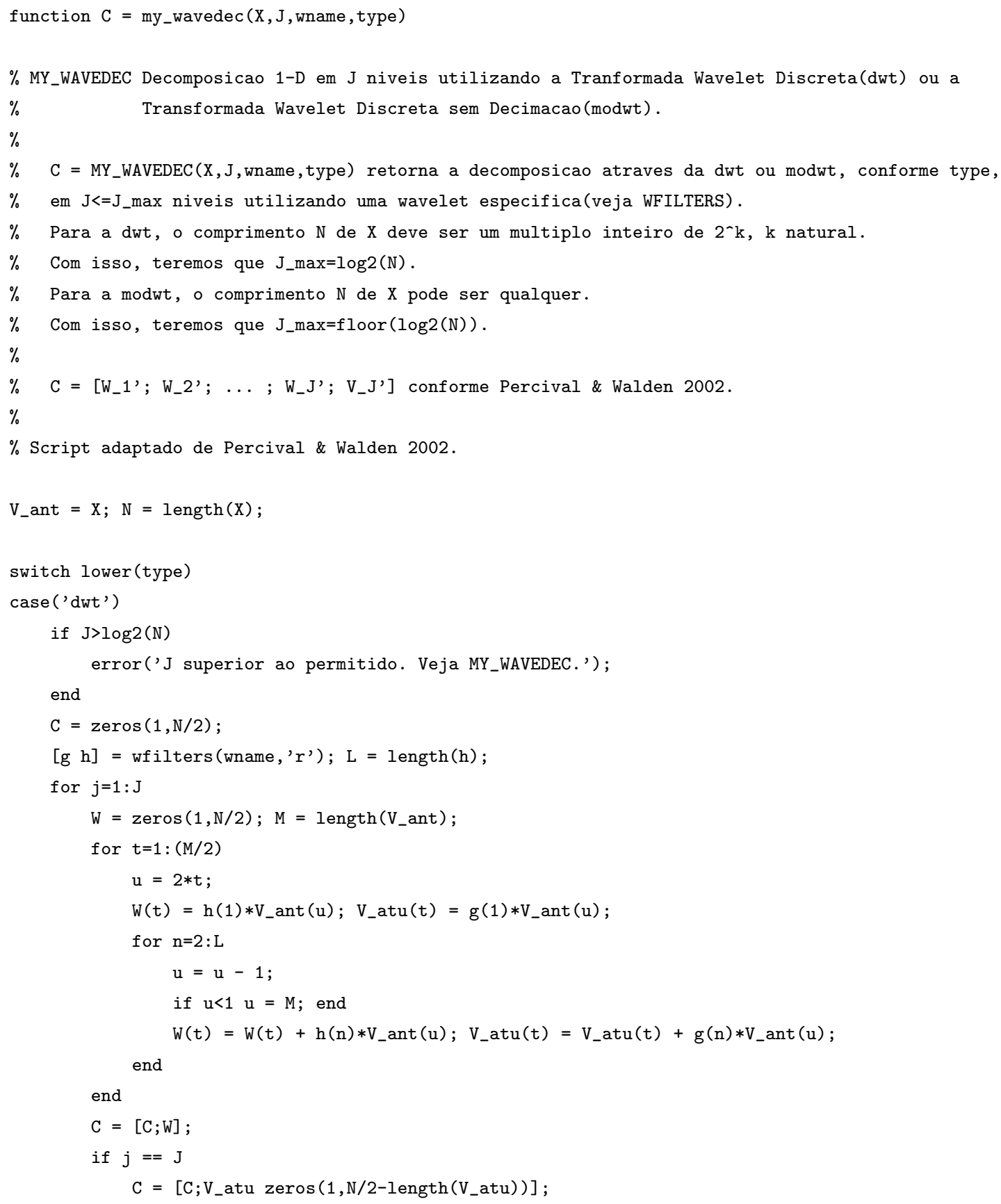




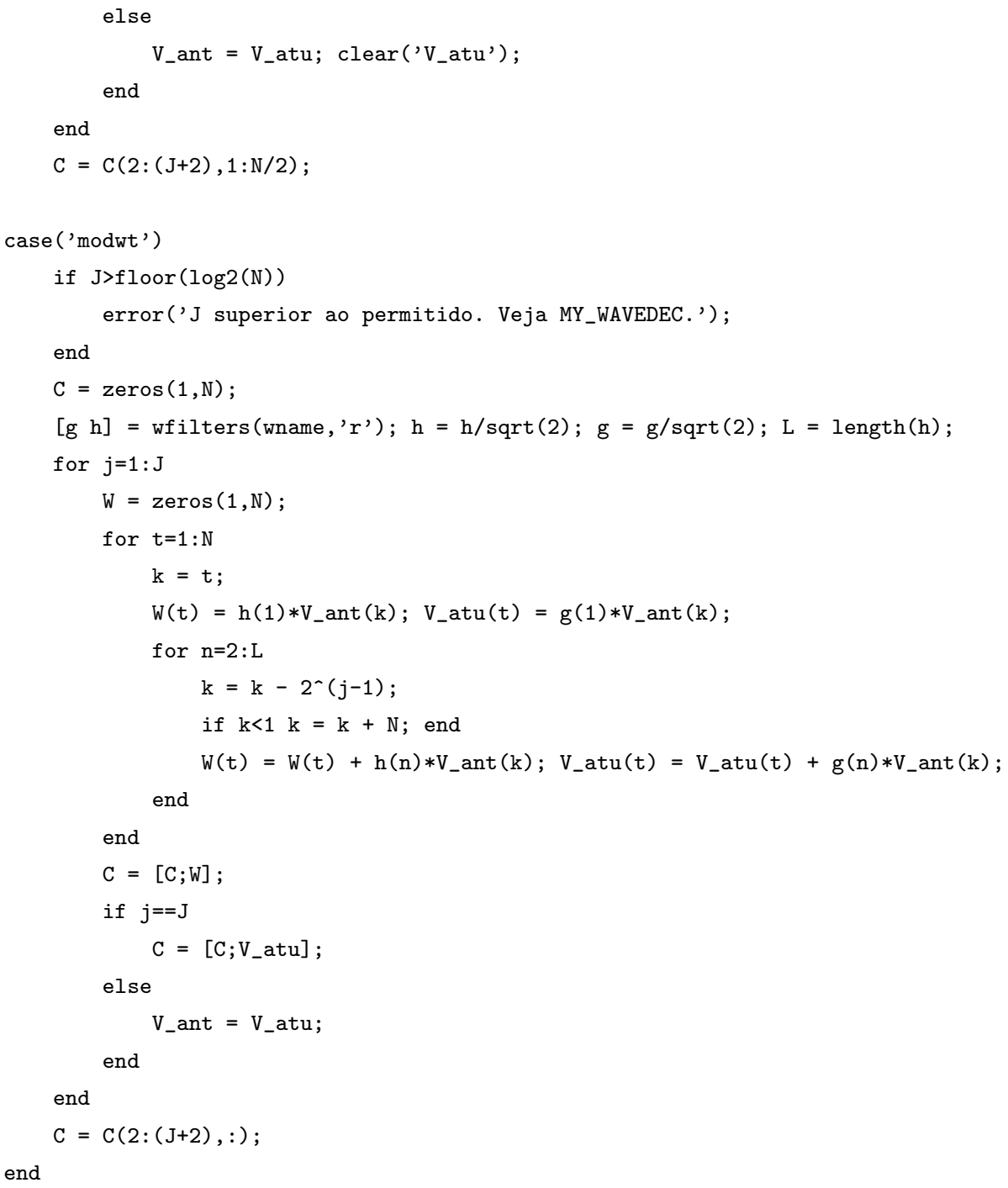




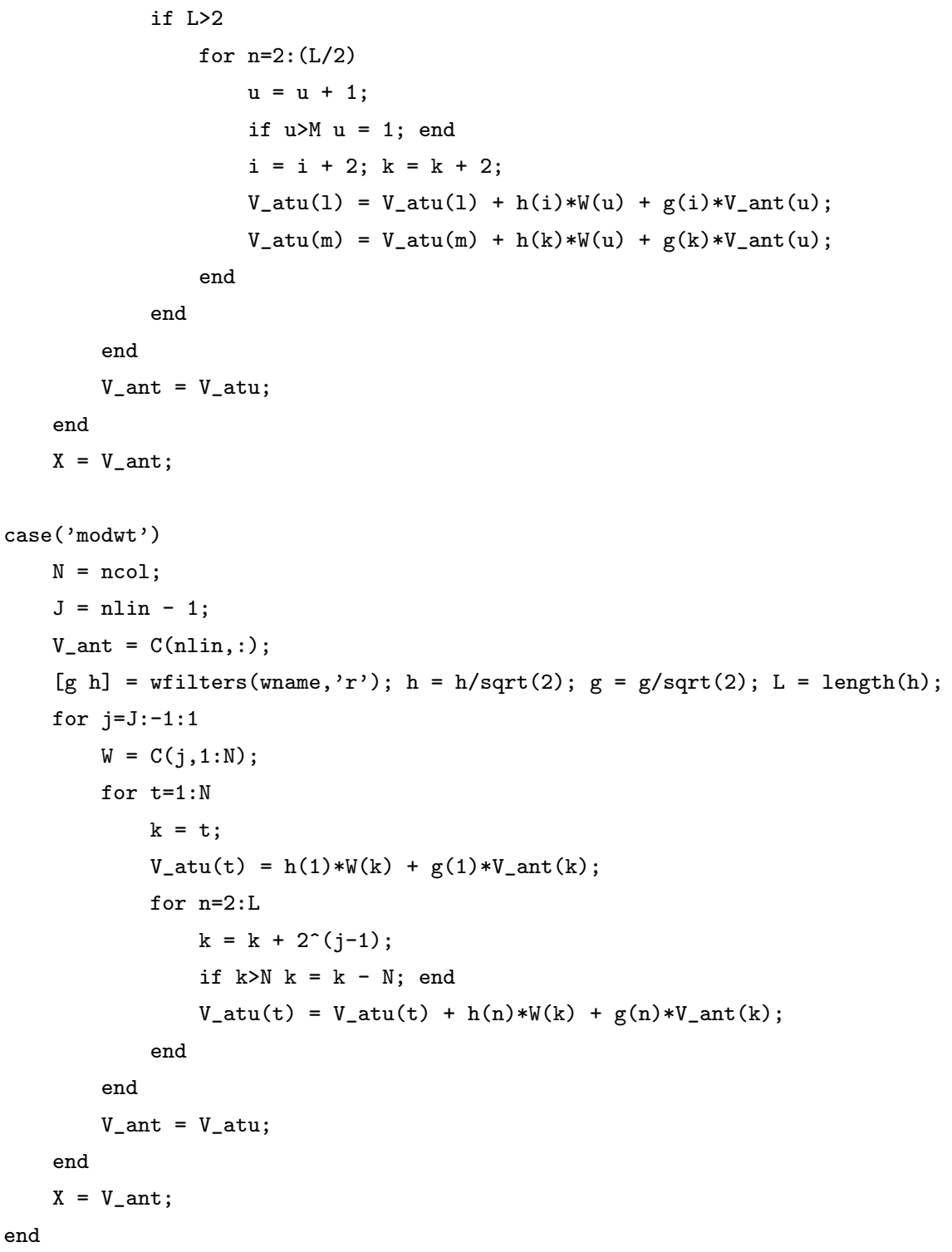




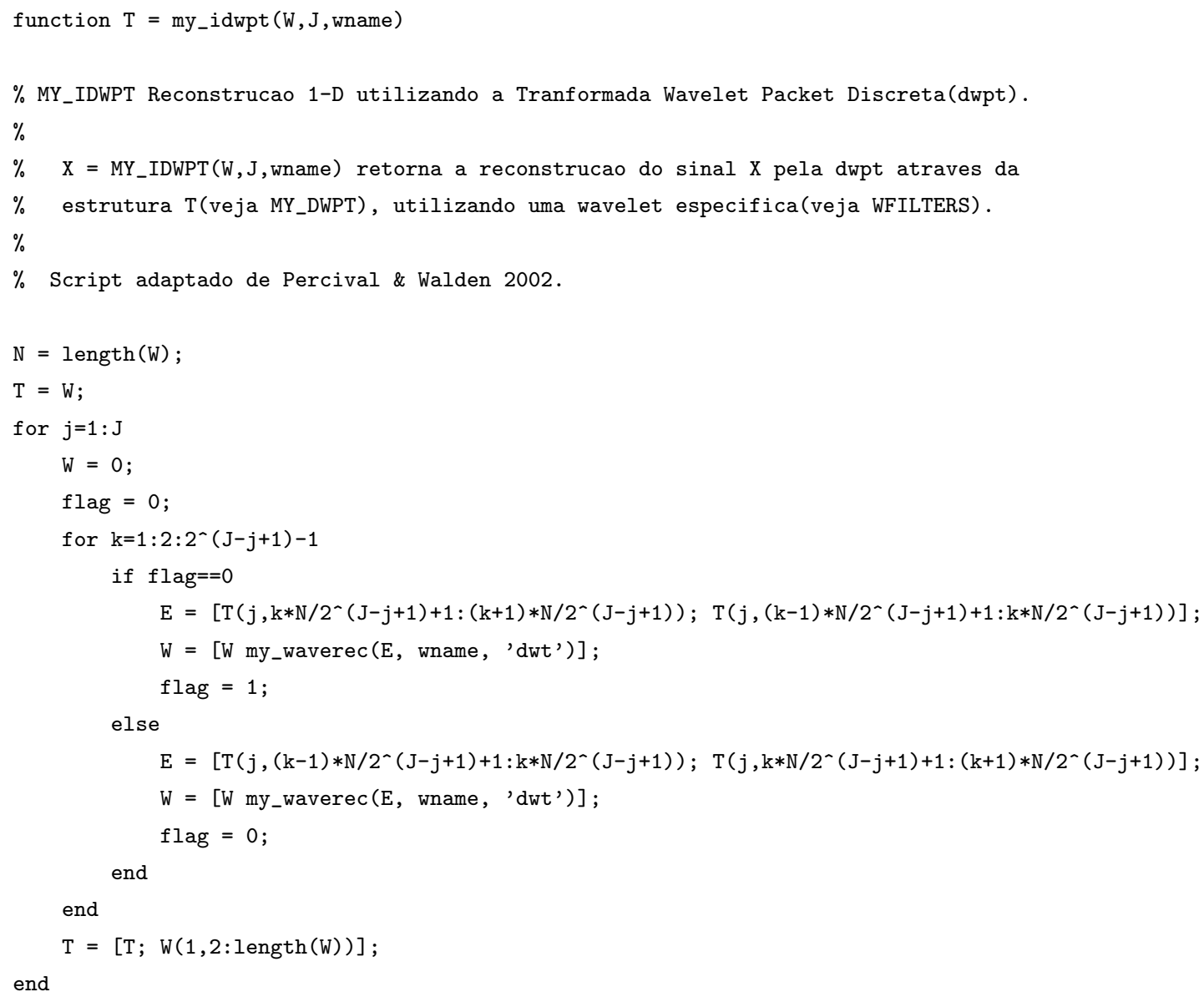




\section{Referências}

[1] EFRON, B. Bootstrap Methods: Another Look at the Jackknife. Annals of Statistics, v. 7, p. 1-26, 1979.

[2] BUllmorE, E. et al. Colored Noise and Computational Inference in Neurophysiological Time Series Analysis: Resampling Methods in Time and Wavelet Domains. Human Brain Mapping, v. 12, p. 61-78, 2001.

[3] BACCALA, L. A.; TAKAHASHI, D. Y.; SAMESHIMA, K. Computer Intensive Testing for the Influence Between Time Series. In: SCHELTER, B.; WINTERHALDER, M.; TIMMER, J. (Ed.). Handbook of Time Series Analysis: Introduction and Overview. [S.l.]: WILEY-VCH Verlag GmbH \& KGaA, 2006 .

[4] MALlAT, S. G. A Theory for Multiresolution Signal Decomposition: The Wavelet Representation. IEEE Transactions on Pattern Analysis and Machine Intelligence, v. 11, p. 674-693, 1989.

[5] FENG, H.; WILLEMIAN, T.; SHANG, N. Wavelet-Based Bootstrap for Time Series Analysis. Communications in Statistics - Simulation and Computation, v. 34, p. 393-413, 2005.

[6] FLANDRIN, P. Wavelet Analysis and Synthesis of Fractional Brownian Motion. IEEE Transactions on Information Theory, v. 38, p. 910-917, 1992.

[7] TEWFIK, A. H.; KIM, M. Correlation Structure of the Discrete Wavelet Coefficients of Fractional Brownian Motion. IEEE Transactions on Information Theory, v. 38, p. 904-909, 1992.

[8] ZHANG J. WALTER, G. A Wavelet-Based KL-Like Expansion for WideSense Stationary Random Processes. IEEE Transactions on Signal Processing, v. 42, p. 1737-1745, 1994.

[9] LIANSHENG, T.; WOODWARD, W. A.; SCHUCANY, W. R. Undercoverage of Wavelet-Based Resampling Confidence Intervals. Communications in Statistics - Simulation and Computation, v. 37, n. 6-7, p. 1307-1315, 2008.

[10] BREAKSPEAR, M. et al. Spatiotemporal Wavelet Resampling for Functional Neuroimaging Data. Human Brain Mapping, v. 23, p. 1-25, 2004.

[11] PERCIVAL, D. B.; WALDEN, A. T. Wavelet Methods for Time Series Analysis. New York: Cambridge University Press, 2000. (Cambridge Series in Statistical and Probabilistic Mathematics).

[12] HALL, P. The Bootstrap and Edgeworth Expansion. [S.l.]: Springer, 1992. (Springer Series in Statistics). 
[13] EFRON, B.; TIBSHIRANI, R. An Introduction to the Bootstrap. London: Chappman \& Hall, 1993. (Monographs on Statistics and Applied Probability).

[14] ZOUBIR, A. M.; BOASHASH, B. The Bootstrap and its Application in Signal Processing. IEEE Signal Processing Magazine, 1998.

[15] ZOUBIR, A. M.; ISKANDER, D. R. Bootstrap Techniques for Signal Processing. Cambridge: Cambridge University Press, 2004.

[16] BROCKWELL, P. J.; DAVIS, R. A. Time Series: Theory and Methods. 2nd. ed. [S.l.]: Springer, 1998.

[17] KEnDALL, M. G.; STUART, A.; ORD, J. K. Advanced Theory of Statistics. 4nd. ed. London: Charles Griffin, 1983.

[18] REGAliA, P. A. Adaptive IIR Filtering in Signal Processing and Control. New York: Marcel Dekker, 1995.

[19] FRIMAN, O.; WESTIN, C. F. Resampling fMRI Time Series. NeuroImage, v. 25, p. 859-867, 2005.

[20] LAIRD, A. R.; ROGERS, B. P.; MEYERAND, E. Comparison of Fourier and Wavelet Resampling Methods. Magnetic Ressonance in Medicine, v. 51, p. 418-422, 2004.

[21] LAHIRI, S. N. Resampling Methods for Dependent Data. New York: Springer, 2003. (Springer Series in Statistics).

[22] LUTKEPOHL, H. Introduction to Multiple Time Series Analysis. Berlim: Springer-Verlag, 1991.

[23] VAIDYANATHAN, P. P. Multirate Systems and Filter Banks. New Jersey: Prentice Hall, 1993.

[24] AKANSU, A. N.; HADDAD, R. A. Multiresolution Signal Decomposition: Transforms, Subbands and Wavelets. San Diego: Academic Press, 2001.

[25] DAUBECHIES, I. Ten Lectures on Wavelets. Philadelphia: SIAM, 1992. (CBMS-NSF Regional Conference Series in Applied Mathematics, v. 61).

[26] STOICA, P.; MOSES, R. L. Introduction to Spectral Analysis. Upper Saddle River: Prentice Hall, 1997.

[27] PRIESTLEY, M. B. Spectral Analysis and Time Series. London: Academic Press, 1982.

[28] KAY, S. M. Modern Spectral Estimation: Theory and Application. New Jersey: Prentice Hall, 1988.

[29] LAHIRI, S. N. Sieve Bootstrap with Variable-Length Markov Chains for Stationary Categorical Time Series. Journal of American Statistical Association, v. 97 , p. 460-462, 2002.

[30] POLITIS, D. N. The Impact of Bootstrap Methods on Time Series Analysis. Statistical Science, v. 18, p. 219-230, 2003.

[31] VIDAKOVIC, B. Statistical Modeling by Wavelets. New York: John Wiley \& Sons, Inc, 1999. 
Apêndice A - Artigo Publicado 